\title{
Distribución geográfica de la avifauna en la Sierra Madre Oriental de San Luis Potosí, México: un análisis regional de su estado de conservación
}

\author{
Francisco Javier Sahagún Sánchez ${ }^{1}$, Jaime Castro Navarro ${ }^{2}$ \& Humberto Reyes Hernández ${ }^{3}$
}

1. Programa Multidisciplinario de Posgrado en Ciencias Ambientales, Coordinación de Ciencias Sociales y Humanidades, Universidad Autónoma de San Luis Potosí, Av. Industrias No 101-A, Fracc. Talleres. C.P. 78494, San Luis Potosí; momotus@gmail.com

2. Museo de Zoología "Alfonso L. Herrera", Facultad de Ciencias, Universidad Nacional Autónoma de México. Cd. Universitaria, Apartado Postal 70-399, México D. F. 04510, México; jaime_118@yahoo.com.mx

3. Coordinación de Ciencias Sociales y Humanidades, Universidad Autónoma de San Luis Potosí, Av. Industrias N ${ }^{\circ}$ 101A, Fracc. Talleres, C.P. 78494, San Luis Potosí; hreyes@uaslp.mx

Recibido 21-III-2012. Corregido 10-IX-2012. Aceptado 02-X-2012.

\begin{abstract}
Geographic distribution of birds in the Sierra Madre Oriental of San Luis Potosí, México: a regional analysis of conservation status. The Sierra Madre Oriental region in the mexican state of San Luis Potosí is a relevant place for bird conservation at a country level. Therefore the main goal of this study was to analyze the geographic patterns of distribution and the conservation current state of the birds, to support the needs to expand the conservation areas in the future. Data was collected from various databases of zoological museums and collections, and field sampling methods conducted from January 2009 to May 2011. Potential distributions were modeled for 284 species using GARP software and then a map was developed to determine areas with favorable environmental characteristics for the distribution of species richness. Finally, the importance of conservation areas for the potential distribution of birds in the region was evaluated. A total of 359 species were recorded of which $71.4 \%$ are permanent residents, $19 \%$ are winter migrants and $4 \%$ are summer residents. From this total, 41 species were endemic, 47 were species at risk and 149 were neotropical migrants. The largest species richness correspond to oak forests, cloud forests, and tropical moist forests located at altitudes from $100 \mathrm{~m}$ to $1500 \mathrm{~m}$. Their potential distribution was concentrated towards the center and Southeast of the study area. Only $10 \%$ of areas with a high potential conservation was included in areas of priority for bird conservation (AICA) and just $3 \%$ of all potential areas were under some governmental category of protection. However, no conservation area has a management plan currently applied and monitored. The information generated is important for the development of management proposals for birds conservation in the region. Rev. Biol. Trop. 61 (2): 897-925. Epub 2013 June 01.
\end{abstract}

Key words: distribution, birds, niches, GARP, conservation, Sierra Madre Oriental, México.

A nivel global los problemas relacionados con la pérdida de la biodiversidad han impulsado el desarrollo de diversas políticas públicas de conservación en muchos países, principalmente en los trópicos, donde la intensidad de los cambios en el uso de suelo y la fragmentación es acelerada y ha causado una palpable disminución de la riqueza y densidades poblacionales de animales y plantas. Lo anterior, ha tenido efectos en términos de servicios ambientales y requiere de acciones inmediatas (Mas et al. 2002, Lampila et al. 2005, Reyes et al. 2008).

Este es un tema prioritario para México, ya que el país está considerado como uno de los más biodiversos a nivel global (Mittermeier 1988, Ramamoorthy et al. 1993, Toledo \& Ordóñez 1998) pero también tiene una de las tasas más altas de deforestación (FAO 2002). En los años recientes se ha incrementado el 
interés por profundizar los estudios para reconocer con mayor detalle los recursos naturales y la biodiversidad asociada a los ecosistemas presentes, particularmente en las regiones que conforman sus sistemas montañosos (OrtegaHuerta \& Peterson 2004, Vargas-Canales 2006, Bird Life International 2008).

La región de la Sierra Madre Oriental (en adelante SMO) es considerada de gran valor e interés para la conservación ya que por encontrarse en una zona de transición entre las regiones Neártica y Neotropical ha generado un mosaico de ambientes en los que se desarrollan niveles altos de diversidad, riqueza y endemismo. En esta región, se distribuyen cerca de 200 especies de reptiles y anfibios, 200 de mamíferos y más de 2500 plantas vasculares (Luna et al. 2004). Asimismo, se ha documentado la presencia de más de 300 especies de aves de las cuales al menos 40 son endémicas (Navarro et al. 2004).Sin embargo, se estima que en los últimos 20 años se desmontaron en la región cerca de 35000 ha de selvas tropicales y de bosques templados (Reyes et al. 2009), lo que representa una tasa anual de deforestación del $2 \%$. Esto, sin duda, ha tenido consecuencias en la calidad y cantidad de hábitats presentes y pone en riesgo la permanencia de zonas que son consideradas importantes para la conservación de las aves y la biodiversidad en general.

Las aves, constituyen uno de los grupos más importantes dentro de la fauna mexicana y a pesar de que existen esfuerzos a nivel estatal y regional para conocer sus patrones de distribución y riqueza, la información generada hasta el momento se considera aún incompleta (Navaro et al. 2004, Vargas-Canales 2006, Palomera-García et al. 2007, Sánchez-González \& García-Trejo 2010, Castro-Navarro 2011).

En el contexto de la heterogeneidad ambiental presente en las regiones tropicales, es necesario el entendimiento de los patrones generales a nivel de especies y comunidades. Sin embargo, la falta de recursos y la escasez de información hacen difícil evaluar a detalle los fenómenos ecológicos e históricos que determinan o afectan dicha distribución (Anderson et al. 2002). Ante este escenario, el uso de los modelos de distribución potencial de nicho constituyen una alternativa eficiente para generar mapas de los sitios con características ambientales similares a las requeridas por las especies (Anderson et al. 2002, Ortega-Huerta \& Peterson 2008, Kumara et al. 2009)

La modelación de nichos ecológicos ha demostrado flexibilidad y conveniencia para maximizar los recursos disponibles en el estudio de la distribución de especies en áreas con extensiones grandes, como es el presente caso de estudio. Existen distintos programas de cómputo de modelación y cada uno utiliza algoritmos matemáticos robustos para realizar las proyecciones; algunos de los más usados son Bioclim (Nix 1986), GARP (Stockwell \& Peters 1999, Peterson 2001) y Max Ent (Phillips et al. 2006). En el presente estudio se eligió usar Desktop GARP (Scachetti-Pereira 2001) con base en los resultados obtenidos en investigaciones previas en sistemas montañosos (Ortega-Huerta \& Peterson 2004, Vargas-Canales 2006).GARP ha sido utilizado con éxito para modelar áreas de distribución geográfica, escenarios ante el cambio climático, áreas de expansión de especies exóticas, estimaciones de daños por plagas a la agricultura, dispersión de enfermedades y diseño de estrategias de priorización para conservación biológica, entre otros (Peterson et al. 2002, Illoldi-Rangel et al. 2004, Elith et al. 2006, Ferrier \& Guisan 2006).

Los objetivos de este estudio incluyeron la realización de un inventario y la compilación de la información existente sobre los registros de la avifauna para analizar los patrones de su distribución geográfica y potencial en la porción de la SMO en el estado de San Luis Potosí. Se comparó la riqueza de las especies por tipo de vegetación y gradientes altitudinales, y se determinó su importancia en el contexto del interés de organismos nacionales e internacionales para la protección de aves en categoría de riesgo por extinción y comercialización, así como por su estatus migratorio. Por último, se generaron mapas de distribución potencial con el programa Desktop GARP para identificar a nivel regional los centros de concentración de la riqueza de especies y evaluar 
la representatividad de las áreas destinadas a la conservación. La información generada es de utilidad en términos de gestión y manejo futuro de los recursos.

\section{MATERIALES Y MÉTODOS}

Área de estudio: La porción de la SMO del estado de San Luis Potosí se extiende en dirección noroeste - sureste a través de la parte centro y sureste del estado, desde las coordenadas $99^{\circ} 29^{\prime} 47^{\prime \prime} \mathrm{W}-98^{\circ} 49^{\prime} 15^{\prime \prime} \mathrm{W}$ y $21^{\circ} 12^{\prime} 7^{\prime}$ ' $\mathrm{N}-22^{\circ} 48^{\prime} 40^{\prime \prime} \mathrm{N}$ (Fig. 1). Incluye fracciones pertenecientes a las subregiones fisiográficas de las Sierras y Llanuras Occidentales, el Carso Huaxteco, la Gran Sierra Plegada y las Sierras Transversales (INEGI 2003). Se presenta un gradiente altitudinal de los $100 \mathrm{y}$ hasta los 2 $700 \mathrm{msnm}$ por lo que los climas varían desde los templados subhúmedos hasta los semicálidos $\mathrm{y}$ cálidos subhúmedos con precipitaciones promedio de entre 500 y $3000 \mathrm{~mm}$ (INEGI 2003). Esta variedad climática ha propiciado la presencia de diversos tipos de vegetación, que incluyen bosques de encinos, bosques mesófilos de montaña, selvas bajas caducifolias y medianas subperennifolias, así como pastizales naturales e inducidos y matorrales submontanos. Se presentan también coberturas de vegetación que han sido transformadas en zonas dedicadas principalmente a la agricultura de riego o de temporal y en algunas áreas se presenta vegetación secundaria. Desde el punto de vista hidrológico, en la SMO se marcan dos zonas bien diferenciadas: hacia lo zona suroriental en la región de barlovento, predominan los climas cálido y semicálido, tanto húmedos como subhúmedos, y las abundantes precipitaciones contribuyen al cauce de ríos importantes como el río Santa María, el río Moctezuma, el río Tampaón y algunos cuerpos de agua como El Salto, La Ciénega, el Puente de Dios y el Ojo

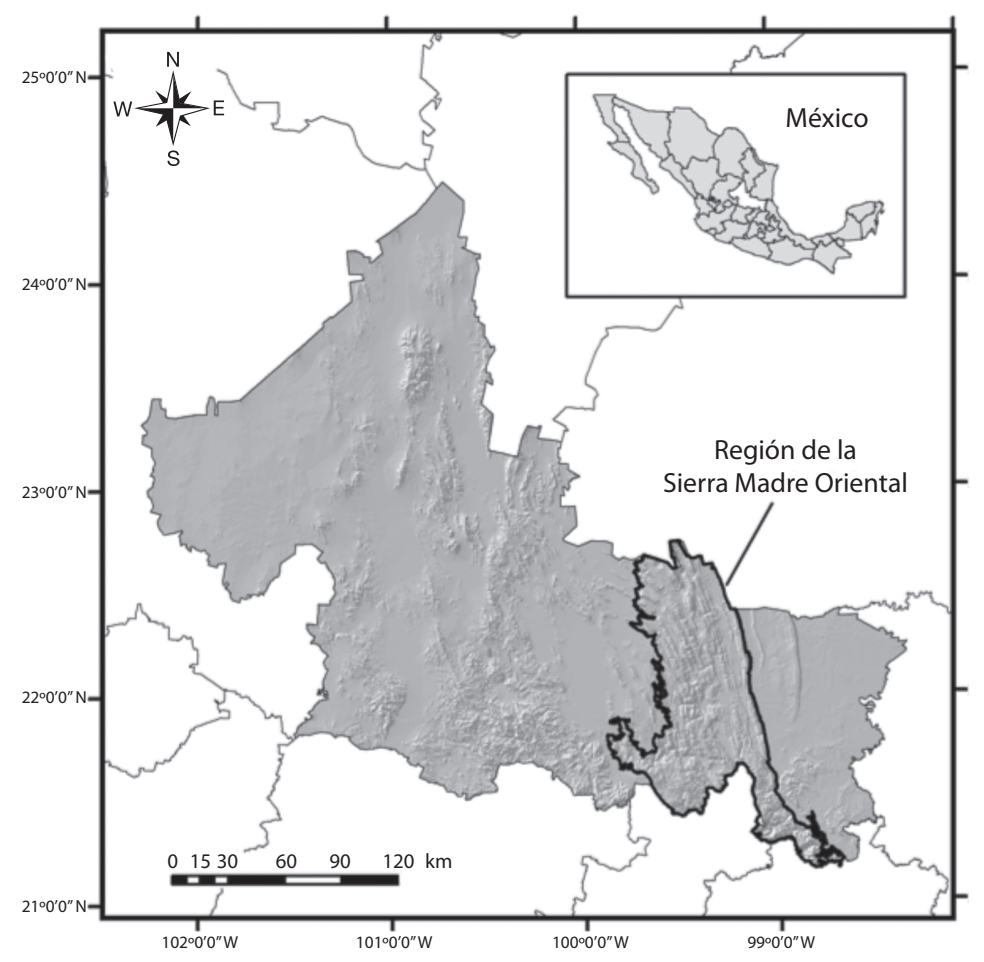

Fig. 1. Sierra Madre Oriental de San Luis Potosí, México.

Fig. 1. Sierra Madre Oriental of San Luis Potosí, México. 
de Agua. Hacia el occidente, en la región de sotavento, predomina el clima seco y semiseco con corrientes de agua de carácter intermitente y de curso reducido (INEGI 2003).

En la región existen seis áreas naturales protegidas (ANP) de carácter estatal que incluyen, El Sótano de las Golondrinas y La Hoya de las Huahuas, con categoría de Monumentos Nacionales; Las Cuevas del Viento y la Fertilidad, catalogada como Sitio Sagrado Natural, la Sierra de En medio, la Sierra del Este y la Reserva Forestal Nacional de la Porción Boscosa del Estado de San Luis Potosí, clasificadas como reservas estatales por su importancia para la conservación. Además, se han delimitado dos sitios RAMSAR (Convención de Humedales): los Arroyos y Manantiales de Tanchachín, localizado en el municipio de Aquismón y la Ciénega de Tamasopo, también conocida como Ciénega de Cabezas, en el municipio de Tamasopo. En su conjunto, estos representan los únicos reservorios con categoría oficial, dedicados a la conservación en esta porción del estado. Existe también un Área de Importancia para la Conservación de las Aves (AICA) denominada San Nicolás de los Montes, ubicada en la confluencia de los municipios de Tamasopo, Alaquines y Cd. del Maíz y reconocida por su alta riqueza avifaunística.

Obtención de datos y análisis de información: Se compiló la información avifaunística a partir de una extensa revisión bibliográfica y de registros en bases de datos de 29 museos y colecciones científicas, y para la que los registros contenidos en la base de datos del Atlas de las Aves de México (Navarro et al. 2003) fueron fundamentales. Se realizó una verificación de todas las localidades con respecto a la cobertura de suelo actual y se depuró el número de registros de las especies de acuerdo con su sensibilidad a las modificaciones en las coberturas y el uso de suelo, basados en Stotz et al. (1996). De esta forma, los registros de especies con sensibilidad alta a las modificaciones, ubicados actualmente en tipos de cobertura antrópicas, fueron excluidas del análisis. Se realizaron ocho salidas de trabajo de campo entre enero del 2009 y mayo del 2011, en sitios de muestreo seleccionados de forma aleatoria en los tipos de cobertura vegetal presentes y en relación a la dinámica de cambio que se determinó en un análisis de modelación espacial previo (Sahagún-Sánchez et al. 2011). Para el levantamiento de la información avifaunística en campo, se combinó el uso de 10 redes de niebla de $12.0 \times 2.5 \mathrm{~m}$ y censos de búsqueda intensiva a través de recorridos en transectos de conteo sin estimar distancia (Ralph et al. 1996), para lo que se utilizaron binoculares 10x50. Las actividades de recolección de datos en redes y censos se realizaron entre las $6: 30 \mathrm{~h}$ y hasta las 11:30h (5h) (Hutto et al. 1986), y se ajustó de acuerdo con el cambio por horario de verano, iniciando a las 5:30h y terminando a las 10:30h. Toda la información obtenida se capturó en una base de datos en formato Access de Microsoft Office para las consultas posteriores. Para la identificación de las especies en campo se utilizaron las guías de Peterson \& Chalif (1989), Howell \& Webb (1995), National Geographic Society (1996) y Van Perlo (2006).

Para el estatus de residencia de las aves, se utilizó la clasificación propuesta por Howell \& Webb (1995) de acuerdo con las siguientes categorías: Residentes permanentes (RB), Residentes de verano (SR), Migratorias residentes de invierno (WV), Transitorias (T) y Otras (O) para especies que presentan poblaciones con combinaciones de las anteriores. Adicionalmente, se determinó el estatus de las especies con respecto al Acta de Conservación de Aves Migratorias Neotropicales (NMBCA) (NMBCA 2011). La categoría de endemismo se estableció de conformidad con los criterios de González-García\& Gómez de Silva (2003) y se clasificó a las especies como endémicas a México, cuasi endémicas o, semiendémicas a México, así como endémicas a la SMO. Asimismo, se les asignó una categoría de riesgo con base en la Norma Oficial Mexicana NOMECOL-059-2010 (DOF 2010), que incluyen "especies sujetas a protección especial" (Pr), "amenazadas" (A) y las que están "probablemente extintas en el medio natural" (P). Además, se analizó el estatus de las especies 
con respecto a la Lista Roja de la Unión Internacional para la Conservación de la Naturaleza (UICN) (UICN 2011) y de la Convención sobre el Comercio Internacional de Especies Amenazadas de Fauna y Flora Silvestre (CITES) (CITES 2011). Finalmente, se elaboró una lista actualizada de las especies (Apéndice 1), basada en la taxonomía propuesta por la American Ornithologists Union (AOU 1998) y los suplementos actualizados al 2011 (http://www.aou. org/checklist/north/print.php).

Se analizó la distribución de las especies por intervalos altitudinales y se aplicó una regresión lineal simple (Zar 1999) para determinar si existía relación entre el número de especies y el gradiente altitudinal. Se estimó la semejanza en la composición de las especies registradas en los distintos tipos de vegetación y uso de suelo con el Índice de Sorensen (IS) en el programa Estimates Ver. 8.2 (Colwell 2011). Este índice se basa en la probabilidad de que dos individuos elegidos al azar de dos muestras diferentes, sean especies compartidas por las dos muestras; el índice en principio es cualitativo y permite determinar la semejanza entre dos muestras al considerar la composición y riqueza de especies de ambas muestras. Cuanto más cercano a 1 sea el valor del índice, mayor semejanza. Para su cálculo se relaciona el número de especies compartidas con la media aritmética de las especies de los sitios comparados. La fórmula aplicada es $\mathrm{IS}=2 \mathrm{~S} / \mathrm{A} 1+\mathrm{B} 1$, donde $\mathrm{S}$ es el número total de especies compartidas, A1 es el número de especies en la cobertura A y $\mathrm{B} 1$ es el número de especies en la cobertura B (Chao et al. 2005). Por último, para probar si existe una diferencia significativa entre el número de especies por tipo de vegetación se aplicó un análisis de varianza (ANOVA) de una vía y posteriormente una prueba de Tukey (Zar 1999) en el paquete estadístico Statgraphics 16 (Statgraphics 2012).

Distribución potencial de nichos y análisis espacial en áreas de conservación: $\mathrm{La}$ modelación de las distribuciones potenciales de nichos, se desarrolló con el programa GARP (Algoritmos Genéticos para generación de
Reglas de Predicción) (Scachetti-Pereira 2001). Este permite predecir la distribución potencial de entidades biológicas a partir de datos geográficos y ambientales en una plataforma de Sistemas de Información Geográfica (SIG) en formato de celdas. El programa es un sistema experto que usa algoritmos genéticos para crear un grupo de soluciones potenciales para el conjunto de datos, a partir de modelos de regresiones logísticas, reglas categóricas, reglas de ámbito o reglas bioclimáticas, y luego, iterativamente, modifica y prueba el conjunto de soluciones hasta que encuentra una solución óptima que es aplicada a los datos de entrenamiento (Ortega-Huerta \& Peterson 2004). El resultado final es la producción de mapas de distribución potencial del nicho ecológico de las especies (Solano \& Feria 2007). Como insumos para el modelo se utilizaron las 19 capas bioclimáticas del World Clim con tamaño de pixel $\sim 1 \mathrm{~km}$ (Hijmanset al. 2005), el modelo de elevación digital de la zona con resolución de 90 x 90m por celda (SRTM 2011), a partir del cual se derivó información sobre la pendiente y las topoformas. Asimismo, se incluyó información sobre las coberturas vegetales y de uso de suelo, obtenida de la interpretación y clasificación supervisada de una imagen Landsat TM del 2005 con resolución de 30m (Reyes et al. 2009). Las capas fueron desplegadas en el SIG ArcMap 9.3 (ESRI 2008), y fueron remuestreadas con un tamaño de pixel de $30 \mathrm{x}$ 30m con proyección WGS_1984_UTM_14N y Datum D_WGS_1984. Los sitios con registros fueron separados en datos de entrenamiento, $(75 \%)$ a partir de los cuales se generaron los modelos y en datos de validación (25\%) usados para la evaluación y prueba interna de precisión en la predicción (Anderson et al. 2003).

Se consideraron las distribuciones potenciales de nicho para 284 especies del total registradas, debido a que contaban con más de cinco puntos de muestreo independientes y a que los modelos resultaron estadísticamente significativos al ser evaluados $\left(\mathrm{x}^{2}, \mathrm{p}<0.05\right)$. Esta prueba permite determinar si los puntos de validación caen en las regiones de presencia predicha con más frecuencia de lo esperado al azar, dada la 
proporción de píxeles predichos en el mapa por el modelo (Anderson et al. 2003). Se corrieron 100 réplicas para cada especie y se eligieron los diez mejores modelos (best subsets). Se estableció como parámetro para la elección de los modelos que no tuvieran errores de omisión $(0 \%)$ y se ubicaran en un umbral del $50 \%$ en la distribución de los errores de comisión (Anderson et al. 2003, Solano \& Feria 2007). Los mejores modelos se sumaron con un umbral de coincidencia de los pixeles con presencia del $75 \%$ o más a través de la herramienta "raster calculator" del módulo de análisis espacial en el SIG, para obtener un mapa de consenso final para cada especie (Illoldi-Rangel et al. 2004, Ríos-Muñoz \& Navarro-Sigüenza 2009). Finalmente, se elaboraron mapas de distribución general de la riqueza mediante la suma de los mapas de todas las especies modeladas.

Para evaluar las extensiones de superficie con alta riqueza de especies, donde tiene un efecto positivo la existencia de áreas dedicadas a la conservación, se realizó una sobreposición de las capas con los polígonos de las ANP's, los sitios RAMSAR y el AICA presentes, sobre el mapa de la riqueza total y se extrajo la información a través de operaciones de recorte en el SIG ArcMap 9.3 (ESRI 2008), la información tabular obtenida se utilizó para calcular las superficies de nichos potenciales, así como el estimado de especies con distribución potencial para cada uno de los polígonos resultantes.

\section{RESULTADOS}

Patrones generales de distribución de la avifauna: Se recolectaron 5002 registros de distribución puntual de 357 especies de aves para la porción de la SMO estudiada. Se encontró que la riqueza avifaunística está compuesta por 20 órdenes y 53 familias. Los órdenes mejor representados fueron el de los Passeriformes con 25 familias y 219spp., lo que equivale al $61 \%$ de la avifauna total, seguido del orden de los Apodiformes con dos familias y con 22spp., correspondiente al $6 \%$ de la riqueza total y los Accipitriformes también con dos familias y $17 \mathrm{spp}$. $(5 \%$ de la riqueza registrada). Dentro del orden de los Passeriformes, las familias con mayor número de especies fueron Parulidae con 38spp. seguida de Tyrannidae con 36spp. y Emberizidae con $24 \mathrm{spp}$. Con respecto a su estacionalidad se encontró que el $71.42 \%$ de las especies son residentes permanentes (RB) (255spp.), el $19 \%$ (68spp.) son aves migratorias de invierno (WV), el 5.32\% (19spp.) son transitorias (T); $3.92 \%$ (14spp.) son residentes de verano (SR) y el $0.2 \%$ restante (1spp.) corresponde a una especie introducida (Apéndice 1).

Del total de especies, 41 presentaron estatus de endemismo, 12 de las cuales son endémicas estrictas de México, 16 son semiendémicas, 11 cuasiendémicas y sólo dos, Dendrortyx barbatus y Glaucidium sanchezi, son endémicas de la SMO (González-García \& Gómez de Silva 2003). De acuerdo con las categoría de riesgo señaladas en la NOM-ECOL-O59-2010 (DOF 2010) se determinó que el $7 \%$ de las especies ( $25 \mathrm{spp}$.) están sujetas a protección especial, el $4 \%$ se consideran amenazadas ( $15 \mathrm{spp}$.) y el $2 \%$ se encuentra en peligro de extinción (8spp.); en esta categoría se incluye a las especies: Amazona oratrix, Amazona viridigenalis, Ara militaris, Cairina moschata, Dendrortyxn barbatus, Geothlypis flavovelata, Glaucidium sanchezi y Spizaetus ornatus. Según UICN (UICN 2011) sólo dos especies se encuentran en situación de peligro de extinción y cuatro son vulnerables. Con respecto a la CITES (CITES 2011) se registraron $49 \mathrm{spp}$. que requieren algún tipo de regulación comercial o protección, y cuatro que se encuentran consideradas en peligro de extinción. De acuerdo al NMBCA (NMBCA 2011), se determinó que 149spp. están enlistadas como migrantes neotropicales y $22 \mathrm{spp}$. son objeto de preocupación para su conservación (Apéndice 1).

El análisis de regresión entre cotas altitudinales y la riqueza reflejó una disminución en el número de especies conforme se incrementa la elevación $\left(\mathrm{R}^{2}=0.807\right)$. El mayor número de especies (274spp.) se registró en la cota altitudinal de entre los $0 \mathrm{~m}$ y los $500 \mathrm{~m}$, de las cuales 110 spp.son consideradas migrantes neotropicales. En este intervalo se registró el 
CUADRO 1

Riqueza de aves por intervalos altitudinales. NMBCA indica las especies con programas de conservación activos actualmente

TABLE 1

Birds species richness by altitudinal intervals. NMBCA indicates species with currently active conservation programs

\begin{tabular}{cccccccc} 
& Riqueza & $\begin{array}{c}\text { Migrantes } \\
\text { neotropicales }\end{array}$ & NMBCA & Endemismo & Categoría de riesgo & IUCN & Cites \\
0 a 500 & 274 & 110 & 13 & 27 & 36 & 26 & 8 \\
500 a 1000 & 253 & 103 & 13 & 29 & 26 & 96 & 38 \\
1000 a 1500 & 235 & 85 & 10 & 30 & 10 & 5 & 34 \\
1500 a 2000 & 118 & 42 & 2 & 16 & & 3 & 10 \\
\hline
\end{tabular}

número más alto de especies que se encuentran en alguna categoría de riesgo por la NOMECOL-059-2010 con 36spp. y de listadas en CITES (46spp.) (Cuadro 1). Asimismo, entre los $500 \mathrm{~m}$ y $1000 \mathrm{~m}$ se determinaron valores altos de riqueza (253spp.), donde resaltan las especies endémicas (29spp.) y las especies migratorias con prioridad para su conservación (13spp.) (Cuadro 1).

Riqueza y semejanza de la composición de especies entre tipos de vegetación y usos de suelo: El ANOVA aplicado mostró que sí existe un efecto del tipo de vegetación sobre el número de especies $(\mathrm{F}=2.21, \mathrm{p}=0.0171)(\mathrm{p} \leq 0.05)$. La prueba de comparaciones múltiples (Tukey) reveló una diferencia estadísticamente significativa $(p \leq 0.05)$ entre las coberturas vegetales de agricultura de riego y bosque de encino (-9-9375);agricultura de riego y bosque mesófilo de montaña (-10.4375); bosque de encino y selva mediana subperennifolia (-9.2777); bosque de encino y vegetación secundaria (10.6) y entre bosque mesófilo y selva mediana suprerennifolia (-9.2777).Se observó un mayor número de especies en coberturas de bosques de encino (213spp.), selvas bajas caducifolias (172spp.) y selvas medianas subperennifolias (169spp.); a estas coberturas les siguen en riqueza el bosques mesófilo de montaña (153spp.) y el matorral submontano (123spp.). Las zonas de coberturas modificadas tales como la agricultura de temporal (121spp.) y los pastizales inducidos (62spp.) presentan también números altos de riqueza (Cuadro 2).
Aunque un número importante de las especies migratorias se concentró en áreas de agricultura de riego y de temporal, muchas otras utilizan los recursos de los bosques y selvas tropicales así como de los humedales presentes (Cuadro 2).De acuerdo con su categoría estacional, se encontró que en el bosque de encino, el bosque mesófilo y la selva baja caducifolia concentran aproximadamente el $70 \%$ de las especies residentes.

Los bosques de encino y el bosque mesófilo de montaña fueron las coberturas con más representatividad de especies endémicas (25spp. y 19spp. respectivamente) (Cuadro 2). En el bosque mesófilo también se detectó la mayor cantidad de especies en alguna categoría de riesgo en la NOM-ECOL-059-2010 y en la lista Roja de la UICN (22spp. y 7 spp. respectivamente), a pesar de que la extensión de esta cobertura se ha reducido drásticamente en los últimos años. En cuanto a la presencia de especies listadas en el apéndice I y II del CITES, se identificó un alto número de especies consideradas en el en las coberturas de bosque de encino (31spp.), selva baja caducifolia (27spp.) y selva mediana subperennifolia (23spp.) (Cuadro 2). Para las especies catalogadas como migrantes neotropicales y aquellas que son objeto de preocupación para su conservación por la NMBCA, los tipos de vegetación más importantes fueron los bosques de encino, el bosque mesófilo de montaña y las selvas tropicales (Cuadro 2).

Al comparar la composición de las especies entre los tipos de cobertura vegetal y usos 
CUADRO 2

Distribución de la riqueza de aves por tipo de vegetación y usos de suelo

TABLE 2

Distribution of bird species richness by vegetation cover and land use

\begin{tabular}{|c|c|c|c|c|c|c|c|c|c|c|c|}
\hline & $\mathrm{AR}$ & AT & $\mathrm{AU}$ & $\mathrm{BE}$ & BMM & MS & $\mathrm{PC}$ & PI & SBC & SMS & VS \\
\hline Riqueza & 47 & 121 & 32 & 213 & 153 & 123 & 27 & 62 & 172 & 169 & 27 \\
\hline$\%$ del total & 13.1 & 33.8 & 8.9 & 59.6 & 42.8 & 34.4 & 7.5 & 17.3 & 48.1 & 47.3 & 7.5 \\
\hline \multicolumn{12}{|l|}{ Estacionalidad } \\
\hline Residente permanente (RB) & 28 & 76 & 21 & 147 & 108 & 87 & 19 & 47 & 117 & 125 & 22 \\
\hline Migratorio de invierno (WV) & 12 & 34 & 7 & 53 & 40 & 32 & 8 & 14 & 45 & 33 & 5 \\
\hline Residente de verano (SR) & 2 & 5 & 2 & 7 & 2 & 2 & 0 & 1 & 4 & 4 & 0 \\
\hline Transitorias $(\mathrm{T})$ & 0 & 6 & 2 & 7 & 3 & 2 & 0 & 0 & 6 & 7 & 0 \\
\hline \multicolumn{12}{|l|}{ NMBCA } \\
\hline Migrantes Neotropicales & 22 & 55 & 17 & 85 & 52 & 48 & 11 & 23 & 74 & 56 & 7 \\
\hline Bajo preocupación de conservación & 2 & 1 & 0 & 9 & 7 & 4 & 0 & 1 & 7 & 7 & 0 \\
\hline \multicolumn{12}{|l|}{ Endemismo } \\
\hline Endémico (E) & 0 & 2 & 0 & 7 & 8 & 4 & 0 & 4 & 3 & 6 & 1 \\
\hline Endémico a la SMO (ES) & 0 & 0 & 0 & 0 & 1 & 0 & 0 & 0 & 0 & 2 & 0 \\
\hline Semiendémico $(\mathrm{S})$ & 5 & 4 & 0 & 10 & 3 & 8 & 0 & 0 & 4 & 5 & 1 \\
\hline Cuasiendémico (Q) & 1 & 4 & 1 & 8 & 7 & 4 & 1 & 2 & 7 & 5 & 2 \\
\hline Total & 6 & 10 & 1 & 25 & 19 & 16 & 1 & 6 & 14 & 18 & 4 \\
\hline \multicolumn{12}{|l|}{ Categoría de riesgo } \\
\hline Amenazada (A) & 0 & 1 & 0 & 4 & 9 & 1 & 2 & 1 & 5 & 3 & 0 \\
\hline En peligro de extinción $(\mathrm{P})$ & 0 & 0 & 0 & 5 & 4 & 1 & 0 & 1 & 3 & 2 & 0 \\
\hline Sometida a protección especial (PR) & 1 & 4 & 0 & 10 & 9 & 8 & 1 & 3 & 9 & 10 & 1 \\
\hline Total & 1 & 5 & 0 & 19 & 22 & 10 & 3 & 5 & 17 & 15 & 1 \\
\hline \multicolumn{12}{|l|}{ IUCN } \\
\hline En peligro (EN) & 0 & 0 & 0 & 2 & 1 & 1 & 0 & 0 & 2 & 0 & 0 \\
\hline Vulnerable (VU) & 0 & 0 & 0 & 2 & 3 & 0 & 0 & 1 & 0 & 1 & 0 \\
\hline En bajo peligro (NT) & 1 & 1 & 0 & 1 & 3 & 2 & 1 & 0 & 3 & 3 & 0 \\
\hline Total & 1 & 1 & 0 & 5 & 7 & 3 & 1 & 1 & 5 & 4 & 0 \\
\hline \multicolumn{12}{|l|}{ CITES } \\
\hline Apéndice I & 0 & 0 & 0 & 3 & 2 & 1 & 0 & 1 & 3 & 0 & 0 \\
\hline Apéndice II & 4 & 13 & 2 & 28 & 13 & 15 & 2 & 4 & 24 & 23 & 2 \\
\hline Apéndice III & 0 & 1 & 0 & 0 & 0 & 0 & 0 & 0 & 0 & 0 & 0 \\
\hline Total & 4 & 14 & 2 & 31 & 15 & 16 & 2 & 5 & 27 & 23 & 2 \\
\hline
\end{tabular}

AR (Agricultura de riego), AT (Agricultura de temporal), AU (Área urbana), BE (Bosque de encino), BMM (Bosque mesófilo de montaña), MS (Matorral Submontano), PC (Pastizal cultivado), PI (Pastizal inducido), SBC (Selva baja caducifolia), SMS (Selva mediana subperennifolia) y VS (Vegetación secundaria).

de suelo, los valores de semejanza más altos se presentaron entre la agricultura de riego y el bosque mesófilo de montaña con un índice de 0.76 , y entre el matorral submontano y la vegetación secundaria (índice de 0.70).Se encontró también una semejanza alta entre las coberturas de selva mediana subperennifolia y bosque mesófilo de montaña con un índice de 0.61 (Cuadro 3). Por otro lado, el bosque de encino presentó índices de semejanza bajos con respecto a las demás coberturas con valores de entre 0.19 y 0.30 , lo que indica una configuración del ensamble de especies muy específica. La menor semejanza se obtuvo entre la 
CUADRO 3

Matriz de similitud de la composición de especies entre coberturas de vegetación

TABLE 3

Similarity matrix of bird species composition among vegetation covers

\begin{tabular}{|c|c|c|c|c|c|c|c|c|c|c|}
\hline & At & $\mathrm{Au}$ & $\mathrm{Be}$ & $\mathrm{Bmm}$ & Ms & $\mathrm{Pc}$ & $\mathrm{Pi}$ & $\mathrm{Sbc}$ & Sms & Vs \\
\hline $\mathrm{Ar}$ & 0.24 & 0.52 & 0.17 & 0.76 & 0.61 & 0.52 & 0.14 & 0.30 & 0.66 & 0.66 \\
\hline At & & 0.33 & 0.23 & 0.26 & 0.23 & 0.35 & 0.19 & 0.28 & 0.23 & 0.26 \\
\hline $\mathrm{Au}$ & & & 0.35 & 0.56 & 0.49 & 0.47 & 0.19 & 0.39 & 0.54 & 0.53 \\
\hline $\mathrm{Be}$ & & & & 0.23 & 0.22 & 0.30 & 0.20 & 0.19 & 0.24 & 0.19 \\
\hline $\mathrm{Bmm}$ & & & & & 0.61 & 0.57 & 0.17 & 0.43 & 0.61 & 0.65 \\
\hline Ms & & & & & & 0.49 & 0.18 & 0.34 & 0.51 & 0.70 \\
\hline $\mathrm{Pc}$ & & & & & & & 0.19 & 0.39 & 0.52 & 0.51 \\
\hline $\mathrm{Pi}$ & & & & & & & & 0.20 & 0.22 & 0.17 \\
\hline Sbc & & & & & & & & & 0.37 & 0.36 \\
\hline Sms & & & & & & & & & & 0.50 \\
\hline
\end{tabular}

$\operatorname{Ar}$ (Agricultura de riego), At (Agricultura de temporal), Au (Área urbana), Be (Bosque de encino), Bmm (Bosque mesófilo de montaña), Ms (Matorral Submontano), Pc (Pastizal cultivado), Pi (Pastizal inducido), Sbc (Selva baja caducifolia), Sms (Selva mediana subperennifolia) y Vs (Vegetación secundaria).

agricultura de riego y los pastizales inducidos (0.14), cobertura esta última que presentó los valores más bajos de semejanza con el resto.

Modelación de nichos ecológicos: Los modelos de distribución generados mostraron una mayor concentración de nichos potenciales para las especies en el intervalo altitudinal de entre los 500-1500m en la parte central de la región y con orientación hacia el sur y este (Fig. 2). Lo anterior coincide con la distribución de las selvas tropicales, los bosques de encinos y mesófilos de montaña, pero también con las zonas donde se desarrollan con más intensidad las actividades de agricultura de temporal y de riego lo que representa una amenaza mayor para las iniciativas de conservación de la avifauna en la zona.

Los municipios donde se presentaron las condiciones ambientales óptimas para una presencia potencialmente alta de especies (150spp. promedio) fueron los de Tamasopo, Ciudad del Maíz, Alaquines, El Naranjo, Santa Catarina y Aquismón. En general, se calculan al menos 284700 ha donde potencialmente podrían registrarse entre 51 y 125 especies. Con menos extensión (34350ha) se presentaron áreas con nichos que albergarían entre 126 y 150 especies y sólo 5398 ha donde podrían distribuirse hasta 175 especies, principalmente en relictos de selva mediana, selva baja caducifolia y algunos sitios con bosque de encino (Cuadro 4). Se identificó una disminución de nichos potenciales para números elevados de especies hacia la región occidental, donde predominan los matorrales submontanos.

Por tipos de vegetación las áreas más representativas se presentaron en los bosques de encino y las selvas tropicales, con un aproximado de 107914ha, donde se determinaron nichos potenciales para 100 y 150 especies; aunque, por otro lado, se calculó que la agricultura de temporal provee de $46352 \mathrm{ha}$ de superficie con potencial de nicho para el mismo número de especies. Altitudinalmente el intervalo con mayor superficie para esta riqueza de especies fue el de entre los $1000 \mathrm{~m}$ y los $1500 \mathrm{~m}$ con un área de 93667 ha.

Distribución de especies de aves migratorias neotropicales: El $41.5 \%$ de las especies registradas han sido catalogadas como migrantes neotropicales por la NMBCA. De estas especies, 22 son prioritarias para su 


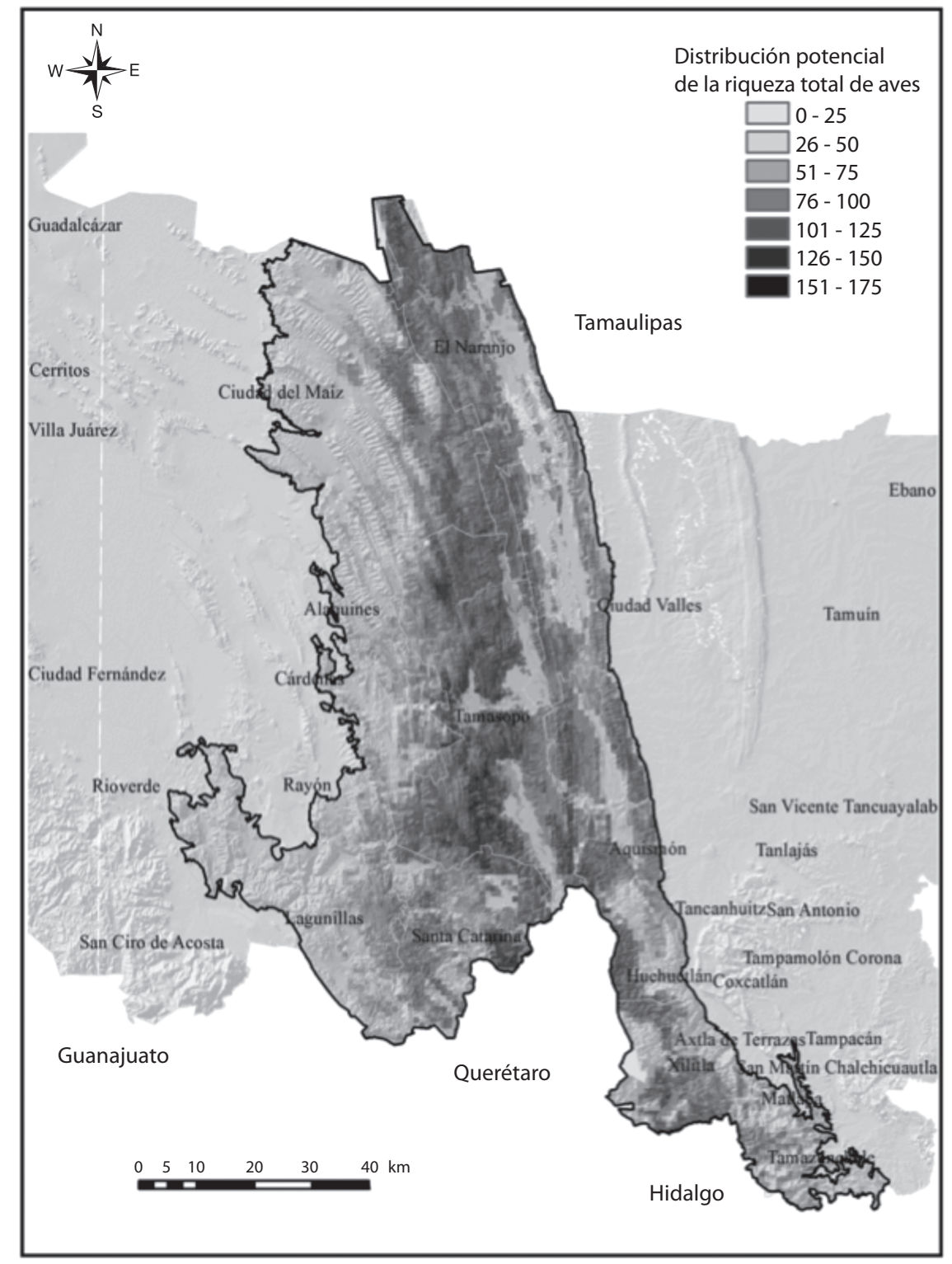

Fig. 2. Distribución potencial de la riqueza total de aves. Las categorías describen el número de especies con nichos potenciales.

Fig. 2. Potential distributions of the birds species richness. The categories describe the number of species with potential niches.

conservación (Apéndice 1) y actualmente existe interés y programas de conservación transnacionales para tal efecto (NMBCA 2011). En el contexto de su distribución potencial, la figura 3 muestra las zonas donde se presentaron los nichos potencialmente ocupados por las especies migratorias bajo preocupación por la NMBCA.

Se pudo apreciar un marcado patrón en la concentración de nichos en áreas con selvas tropicales y bosque de encino, principalmente en los municipios de el Naranjo, Tamasopo y 
CUADRO 4

Área estimada (ha) de distribución potencial de la riqueza de especies en Áreas Naturales Protegidas (ANP), Sitios RAMSAR (Humedales) y Áreas de Importancia para la conservación de las Aves (AICA). Se incluyen porcentajes para cada categoría y totales por áreas dedicadas a la conservación (AC)

TABLE 4

Area estimates (ha) of potential distributions for bird species richness in Natural Protected Areas (ANP's), RAMSAR sites (Wetlands) and areas of priority for bird conservation (AICA). Include percentages and total for conservation areas (AC)

\begin{tabular}{crrrrrrrrr} 
Categoría & $\begin{array}{c}\text { Superficie } \\
\text { de Nichos } \\
\text { potenciales }\end{array}$ & ANPs & $\%$ & $\begin{array}{c}\text { Sitios } \\
\text { RAMSAR }\end{array}$ & $\%$ & AICA & $\%$ & $\begin{array}{c}\text { Superficie } \\
\text { cubierta } \\
\text { por AC }\end{array}$ & $\begin{array}{r}\% \text { total } \\
\text { por AC }\end{array}$ \\
$0-25$ & 234166.05 & 9763.65 & 4.17 & 1350.99 & 0.58 & 13031.73 & 5.57 & 24146.37 & 10.31 \\
$26-50$ & 122448.33 & 2037.96 & 1.66 & 3150.00 & 2.57 & 17280.27 & 14.11 & 22468.23 & 18.35 \\
$51-75$ & 99071.10 & 2703.69 & 2.73 & 1230.21 & 1.24 & 13388.85 & 13.51 & 17322.75 & 17.49 \\
$76-100$ & 147202.83 & 4012.02 & 2.73 & 612.90 & 0.42 & 11988.54 & 8.14 & 16613.46 & 11.29 \\
$101-125$ & 127599.84 & 4028.31 & 3.16 & 15.03 & 0.01 & 13801.86 & 10.82 & 17845.20 & 13.99 \\
$126-150$ & 34347.96 & 70.74 & 0.21 & 3.06 & 0.01 & 3266.28 & 9.51 & 3340.08 & 9.72 \\
$151-175$ & 5398.02 & 0.00 & 0.00 & 0.00 & 0.00 & 554.31 & 10.27 & 554.31 & 10.27 \\
Sup. Total & 770234.13 & 22616.37 & 2.94 & 6362.19 & 0.83 & 73311.84 & 9.52 & 102294.16 & 13.28 \\
\hline
\end{tabular}

Santa Catarina (Fig. 3), distribuidos por abajo de los $1500 \mathrm{~m}$ y cerca de cuerpos de agua, en zonas de borde con tipos de cobertura transformados como la agricultura de riego y de temporal, éstas últimas resultaron importantes como nicho para especies migratorias en general.

Distribución de nichos potenciales en áreas prioritarias para la conservación: Las zonas denominadas como Áreas Naturales Protegidas (ANP) (2.94\%) y los sitios RAMSAR (Convención de Humedales, $0.83 \%$ ), representaron apenas el $3.77 \%$ de la superficie total de la región (Cuadro 4, Fig. 4). En las ANP's se registraron coberturas vegetales de bosques y selvas tropicales, así como agricultura de temporal principalmente, y ocupan una superficie de 22 545ha para nichos potenciales de hasta 125 especies. Muchos de las zonas con riqueza potencial alta no resultaron considerados al interior de las ANP's, a pesar de ser sitios con potencial para implementación de áreas de conservación (ver polígonos A y B en Fig. 4). La Reserva Forestal Nacional de la Porción Boscosa del Estado de San Luis Potosí es la ANP que resultó proporciona la mayor superficie de protección con 20148 ha, de las cuales 7673 ha son aptas para 76 a 125 especies. Por otro lado, los humedales (Sitios RAMSAR) mostraron un aporte importante de nichos por su ubicación espacial y su extensión, además de ser relevantes por el interés que se tiene para su conservación. Las zonas que representaron nichos potenciales para hasta 100 especies alcanzaron las 6362 ha con un mayor aporte de la Ciénega de Tamasopo, donde predominan las coberturas de agricultura de riego y bosques de galería, además de que están en contacto con zonas dominadas por selvas bajas caducifolias.

El área de importancia para la conservación de las aves (AICA) de San Nicolás de los Montes (Fig. 4), con una superficie de 73312ha, presentó la mayor extensión con nichos de distribución potencial y corresponde al $9.52 \%$ de la región estudiada. Las coberturas preponderantes fueron los bosques de encino y algunos relictos de bosque mesófilo de montaña en laderas e incluyeron 17068ha de zonas con nichos potenciales para más de 100 y hasta 150 especies. A diferencia de las ANP's, el AICA albergó 554ha donde potencialmente podrían distribuirse hasta 175 


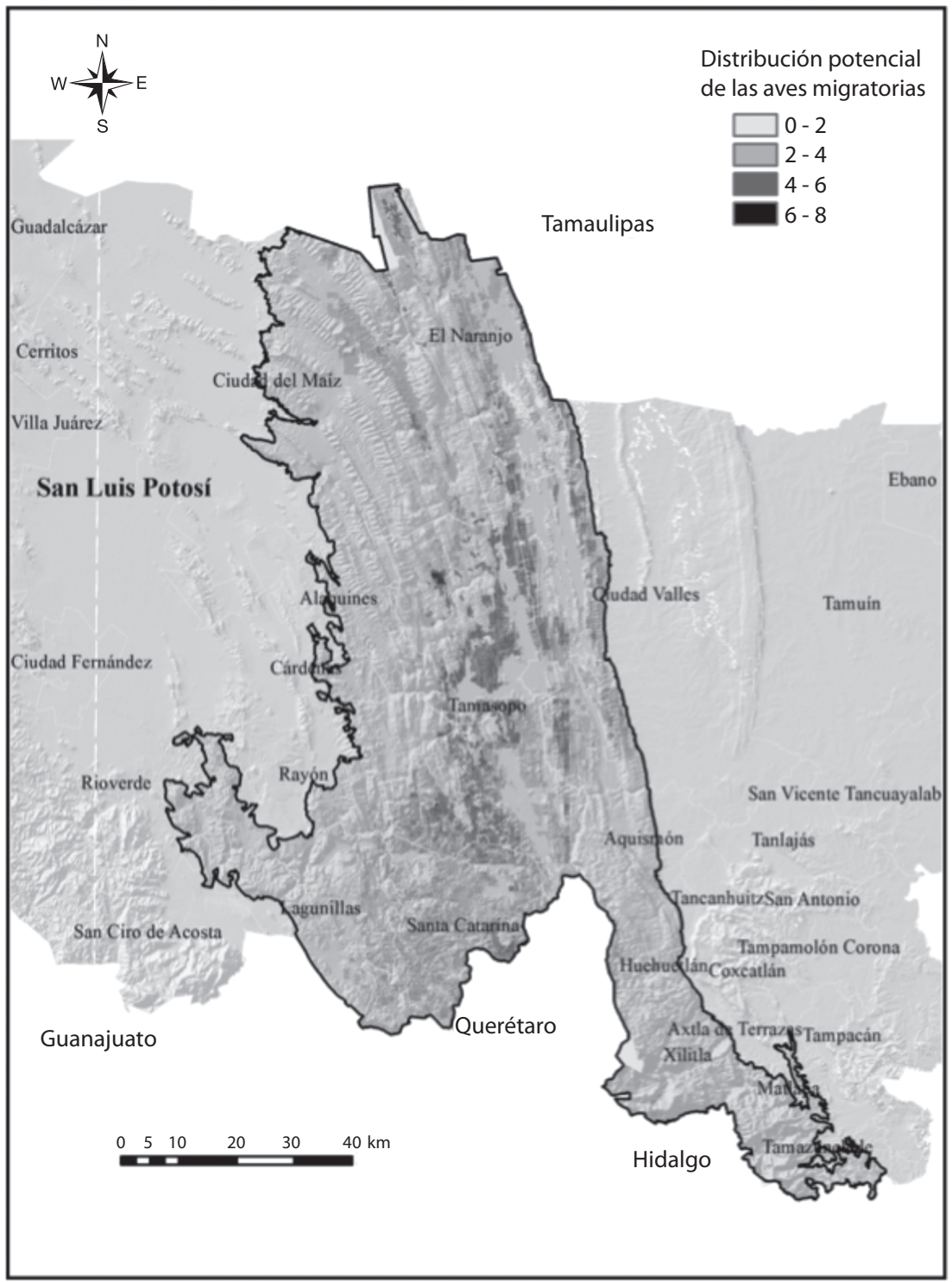

Fig. 3. Distribución potencial de aves migratorias neotropicales de interés para su conservación por la NMBCA. Las categorías describen el número de especies con nichos potenciales.

Fig. 3. Potential distributions of migratory birds under conservation concern by the NMBCA. The categories describe the number of species with potential niches. 


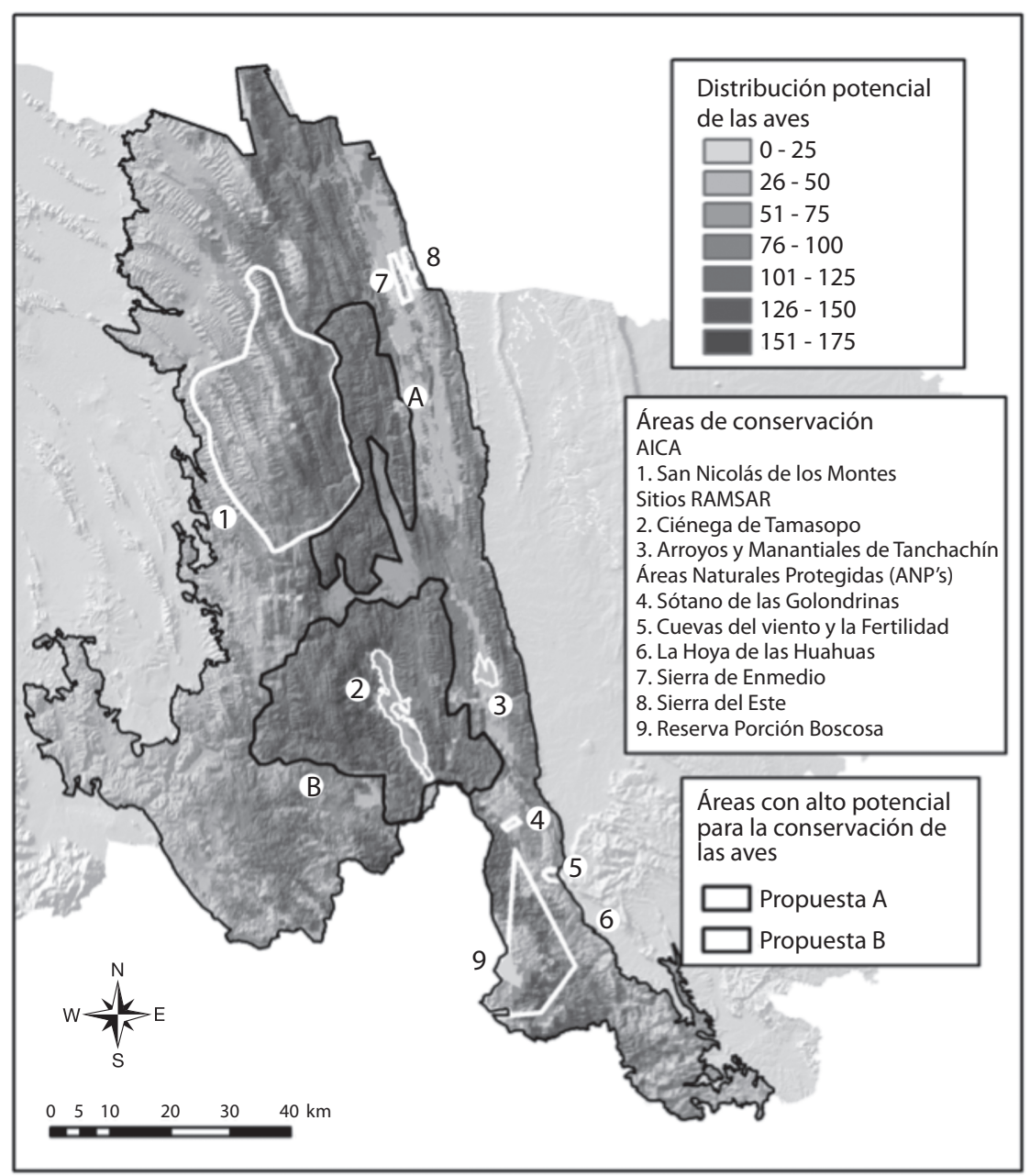

Fig. 4. Representatividad de áreas dedicadas a la conservación con respecto a la distribución potencial de las aves. Se incluyen dos polígonos de áreas con potencial para la conservación.

Fig. 4. Representativeness of conservation areas respect to potential distribution of birds. Includes two polygons of potential areas for conservation.

especies (Cuadro 4); sin embargo, esta región no tiene un estatus oficial como área de conservación a nivel estatal o federal.

\section{DISCUSIÓN}

La riqueza de especies de la SMO de San Luis Potosí corresponde al $68.5 \%$ de las que se estima se encuentra en todo el estado (538spp.). Este número representa el 33\% de la avifauna total registrada para México (1 096spp.) y 3.9\% de las especies en el mundo (Llorente-Bousquets \& Ocegueda 2008). Esta riqueza es mayor incluso que la de algunos estados de México, como Aguascalientes (263spp.) o Guanajuato (350spp.) y es similar a la registrada para Yucatán, Zacatecas, Tlaxcala, Morelos y Coahuila, con 365 especies en promedio (Berlanga et al. 2008). Sin duda, el alto número de especies en relación con la superficie considerada para el estudio confirma la importancia que tiene como punto para la conservación de la biodiversidad 
en el contexto regional y nacional. No obstante, los procesos de cambio en el uso de suelo, con la consecuente transformación y desaparición de hábitats (Sahagún-Sánchez et al. 2011), la fragmentación de los ecosistemas (CastroNavarro 2011), así como la explotación no regulada con fines comerciales y la cacería ilegal, amenazan la permanencia de muchas de las especies en la región. Al respecto, al menos14 especies de aves son aprovechadas con fines ornamentales (INE 1997) aunque en el estado se comercializan sin control rapaces y muchas otras especies canoras.

Por otro lado, el hecho de que más del $70 \%$ de las especies sean residentes indica el elevado número de componentes neotropicales presentes; un patrón consistente con lo registrado a nivel nacional, especialmente en regiones tropicales (Escalante et al. 1993). En el área de estudio y como ha sido descrito para algunas otras regiones del país (Palomera-García et al. 1994) el número de las especies migratorias disminuye hacia el sur. La región es, sin duda, un corredor de migración importante (VillaBonilla et al. 2008), dado el alto número de especies presentes consideradas como migratorias neotropicales. Hacer énfasis en la presencia de las especies migratorias es pertinente dado que muchas de las poblaciones están amenazadas a nivel continental por los cambios en el uso de suelo. Conocer su distribución y el estado de los hábitats en las distintas regiones que ocupan, facilitará la implementación de acciones comunes de conservación a corto y mediano plazo.

A pesar de que la SMO no está considerada como uno de los centros de evolución principales para los táxones en el país (García-Trejo \& Navarro-Sigüenza 2004), la porción estudiada concentra el $11.4 \%$ de las especies endémicas para México, incluyendo especies como Aratinga holochlora, Atlapetes pileatus, Atthis heloisa, Catharus occidentalis y Rodothraupis celaeno; y a diferencia de otras regiones, incluye también dos especies que son endémicas exclusivas (Dendrortyx barbatus y Glaucidium sanchezi). Por otra parte, más del $24 \%$ de las especies listadas en la NOM-ECOL-O59-2010, el UICN y CITES, están representadas en la región, lo que alerta sobre la necesidad de trabajar en la identificación de los sitios con hábitats relevantes para el mantenimiento de estas poblaciones y sobre el monitoreo de las prácticas actuales para su aprovechamiento.

Las coberturas vegetales con la riqueza de especies más alta, se ubican principalmente en la región de barlovento de la sierra, en dirección a la Planicie del Golfo en un gradiente altitudinal que inicia por sobre los $1500 \mathrm{~m}$. Esto coincide con las hipótesis generales de que al ámbito altitudinal, una precipitación y humedad mayor, así como un relieve más abrupto son determinantes para una mayor riqueza avifaunística (Ruggiero \& Hawkins 2008) y coincide con lo consignado en estudios similares (Vargas-Canales 2006, Castro-Navarro 2011). En el caso del bosque de encino, que tiene la riqueza de especies más alta, el análisis de semejanza demostró que las especies que conforman la comunidad, son en su mayoría, diferentes a las existentes en las otras coberturas, y predominan las especies residentes y propias de climas templados; ésta marcada separación, ha sido encontrada en otros estudios en la SMO (Sahagún-Sánchez 2003). Asimismo, presentó junto con el bosque mesófilo, la mayor cantidad de especies endémicas, lo cual coincide con los patrones registrados en otras regiones del país (Villa-Bonilla et al. 2008). El bosque mesófilo es considerado uno de los ecosistemas más biodiversos, además de que provee importantes servicios ambientales (Williams et al. 2002). Se estima que a nivel nacional el $70 \%$ de las especies endémicas de aves se distribuyen en él (FloresVillela \& Gerez 1994) y en el caso de este estudio incluye también la mayor cantidad de especies en categoría de riesgo; sin embargo, en la región se ha documentado la pérdida del $47 \%$ de la superficie en los últimos 34 años, lo que sin duda, ha tenido un fuerte impacto sobre las especies y sus poblaciones originales.

En las selvas tropicales, bajas caducifolias y medianas subperennifolias, el alto número de especies es un patrón recurrente (RamírezAlbores 2010) y está determinado en la SMO 
por su extensión y su complejidad estructural, lo que provee mayor disponibilidad de recursos. Las coberturas modificadas por las actividades humanas muestran también una riqueza de especies elevada; no obstante, muchas de las especies son de hábitos generalistas lo que facilita su adecuación a los cambios inducidos, en detrimento de las poblaciones de especies selectivas (Stotzet al. 1996). En la región de la huasteca existen cultivos de café aledaños a coberturas de selvas y bosques mesófilos, donde se han registrado números altos de especies, lo que les confiere un valor especial como áreas de distribución potencial (Petit \& Petit 2003). También, los humedales de la región tienen una función importante para muchas de las especies migratorias y residentes, esto, debido a su orientación norte sur, y quizá, a que se encuentran en tierras bajas con predominio de áreas de contacto con selvas tropicales donde se concentra mucha de la riqueza de especies. Además de proveer recursos vinculados a la presencia de agua, sirven como reguladores de los microclimas, esenciales para muchas especies residentes, y como zona de descanso y forrajeo para migratorias de hábitos acuáticos; por lo que su pérdida significaría una amenaza para un número importante de especies.

A nivel estatal, la superficie dedicada a la conservación representa el 8\%. En la región ésta superficie apenas alcanza el 3\%; sin embargo, los planes de manejo para las ANP's existentes aún se encuentran en proceso de elaboración o son inexistentes. Las marcadas diferencias en superficie y configuración de las áreas naturales protegidas o propuestas por su importancia para la conservación, determinan que las zonas con alta concentración de nichos estén sólo parcialmente representadas. En este sentido, promover un decreto oficial para que la AICA de San Nicolás de los Montes se convierta en un ANP, significaría incrementar al $13.28 \%$ la superficie bajo algún régimen de conservación, acercándose a cumplir con la cuota sugerida a nivel internacional de contar con al menos el $17 \%$ de la superficie terrestre considerada como área protegida (CBD 2011).
Resulta muy significativo que en la región se presenten extensiones importantes de cuatro coberturas vegetales fundamentales para la conservación de especies migratorias, de interés para distintos países, debido a que son consideradas especies claves e indicadoras del estado de salud de los ecosistemas (Berlanga et al. 2010). Estas coberturas (bosque de encino, bosque mesófilo de montaña, selva baja caducifolia y selva mediana subperennifolia), constituyen un activo natural importante para el mantenimiento de poblaciones de muchas especies, y además son fuente de servicios ambientales para las comunidades y habitantes de las zonas aledañas. Sin embargo, se ha determinado que existe un importante hueco en la representación de estas coberturas vegetales en las ANP de la zona establecidas actualmente, por lo que se deben acelerar los procesos de gestión para lograr su protección (Chapa-Vargas \& Monzalvo-Santos 2012).Por su orientación, la SMO funciona como corredor para muchas de las especies, por lo que la implementación de una iniciativa que permita ampliar la cobertura de las áreas dedicadas a la conservación a nivel regional facilitaría la conectividad con las áreas naturales protegidas ya existentes en la zona y las que se encuentran en los estados adyacentes como son la Reserva de la Biosfera de Sierra Gorda en Querétaro y la Reserva del Cielo en Tamaulipas.

La distribución potencial de nichos ecológicos modelada, provee de información relevante para identificar los centros de riqueza a nivel regional y permite valorar la superficie que debería ser sometida a programas de manejo sustentable, de forma que se garantice la conservación de los hábitat relevantes para el mayor número de especies (Illoldi et al. 2008). A pesar de que los modelos sólo representan hipótesis de la distribución de las especies, su uso es plenamente justificado cuando existen restricciones de tiempo y recursos (Solano \& Feria 2007, Ríos-Muñoz \& Navarro-Sigüenza 2009). Además, el análisis espacial de los nichos y su correspondencia con las áreas dedicadas a la conservación, constituye una herramienta valiosa para el diseño y 
el establecimiento de políticas ad hoc (OrtegaHuerta \& Peterson 2004).

En general, el estudio de la biodiversidad a escala regional, permite abordar la problemática a partir de áreas naturales, de forma que las iniciativas de conservación derivadas tienen mayor congruencia en el contexto del paisaje, los ecosistemas y las ANP's presentes (Ruíz-Jiménez et al. 2004). Cuando se utiliza el formato y la resolución espacial adecuada, es posible moverse a niveles municipales o locales a través de un análisis multi escalar para la implementación de acciones de conservación, con implicaciones importantes en términos de gestión ambiental. Además, las regiones coinciden muchas veces con territorios donde se puede identificar claramente la identidad socio cultural de los habitantes, lo que resulta fundamental para desarrollar los procesos participativos y de apropiación de las iniciativas requeridas para lograr una planeación sistemática para la conservación (Margules \& Pressey 2000).

\section{AGRADECIMIENTOS}

A Adolfo G. Navarro Sigüenza del Museo de Zoología "Alfonso L. Herrera" de la Facultad de Ciencias de la UNAM, por facilitar los datos de la base del Atlas de las Aves de México y a los Museos y Colecciones Zoológicas nacionales e internacionales de las que provienen muchos de los registros. A Francisco Martin Huerta Martínez, Gerardo Sánchez y dos revisores anónimos por sus comentarios y sugerencias para mejorar el documento. Al CONACYT por la beca otorgada para la realización de los estudios de doctorado, convenio 80889 . Proyecto SEMARNATCONACYT: 2006-C01-23754.

\section{RESUMEN}

La región de la Sierra Madre Oriental (SMO) en el estado de San Luis Potosí en México es considerada un sitio de relevancia para la conservación de la avifauna a nivel nacional. Por lo anterior, el objetivo de este estudio fue analizar la distribución geográfica de las aves en la zona para identificar las áreas con mayor concentración de la riqueza y evaluar su estado actual de conservación para fundamentar la necesidad de incrementar la superficie dedicada a las áreas naturales protegidas en el futuro. Se recolectó información proveniente de diversas bases de datos de colecciones y museos, además de realizar trabajo de campo en el periodo de enero 2009 a Mayo 2011. Se modelaron las distribuciones potenciales para 284 especies con el programa GARP y se elaboró un mapa de consenso para determinar la ubicación espacial de las áreas con condiciones ambientales para soportar el mayor número de especies. Finalmente, se evaluó la representatividad de las zonas dedicadas a la conservación para las aves. Se registraron 357 especies, de las cuales el 71.4\% son residentes permanentes, $19 \%$ son migratorias de invierno y $3.9 \%$ residentes de verano. Del total, 41 especies son endémicas y 48 se encuentran en alguna categoría de riesgo; la mayor concentración de especies se presentó en las coberturas de bosques de encino, bosques mesófilos y selvas medianas y bajas caducifolias, en un intervalo altitudinal entre 100-1 $500 \mathrm{~m}$ con una más alta cantidad de nichos en la parte centro y hacia el sur y este de la región. Sólo el 10\% de las zonas con alto potencial de número de especies está incluido como área de importancia para la conservación de las aves y el 3\% del total de áreas potenciales está bajo alguna categoría estatal de protección; sin embargo, ninguna de las áreas tiene un plan de manejo que esté siendo aplicado y monitoreado. La información generada es importante para el desarrollo de propuestas de conservación para las aves en la región.

Palabras clave: distribución, avifauna, nichos, GARP, conservación, Sierra Madre Oriental, México.

\section{REFERENCIAS}

Anderson, R.P., M. Gómez-Laverde \& A.T. Peterson. 2002. Geographical distributions of spiny pocket mice in South America: insights from predictive models. Global Ecol. Biogeogr. 11: 131-141.

Anderson, R.P., D. Lew \& A.T. Peterson. 2003. Evaluating predictive models of species' distributions: Criteria for selecting optimal models. Ecol. Model. 162: 211-232.

AOU. American Ornithologist's Union. 1998. Check-list of North American Birds. Committe on Classification and Nomenclature. EE.UU.

Berlanga, H., V. Rodríguez-Contreras, A. Oliveras de Ita, M. Escobar, L. Rodríguez, J. Vieyra \& V. Vargas. 2008. Red de Conocimientos sobre las Aves de México. (AVESMX). CONABIO. (Consultado: 6 junio 2011, http://avesmx.conabio.gob.mx/).

Berlanga, H., J.A. Kennedy, T.D. Rich, M.C. Arizmendi, C.J. Beardmore, P.J. Blancher, G.S. Butcher, A.R. Couturier, A.A. Dayer, D.W. Demarest, W.E. Easton, 
M. Gustafson, E. Iñigo-Elias, E.A. Krebs, A.O. Panjabi, V. Rodríguez-Contreras, K.V. Rosenberg, J.M. Ruth, E. Santana-Castellón, R. Ma. Vidal \& T. Will. 2010. Conservando a nuestras aves compartidas: La visión trinacional de Compañeros en Vuelo para la conservación de las aves terrestres. Cornell Lab of Ornithology. Ithaca, Nueva York, EE.UU.

Bird Life International. 2008. State of the world's birds. Indicators for our changing world.

Castro-Navarro, J. 2011. Proceso de fragmentación del hábitat en la Sierra Madre Oriental Potosina y su impacto en la distribución de la avifauna. Tesis de Licenciatura, UNAM. México, D.F., México.

CBD. 2011.Convention on Biological Diversity.Aichi Biodiversity Targets. (Consultado: 5 noviembre 2011, http://www.cbd.int/sp/targets/).

Chao, A., R.L. Chazdon, R.K. Colwell \& S. Tsung-Jen. 2005. Un nuevo método estadístico para la evaluación de la similitud en la composición de especies con datos de incidencia y abundancia, p. 85-96. In G. Halffter, J. Soberón, P. Koleff \& A. Melic (eds.) Sobre Diversidad Biológica: El significado de las Diversidades Alfa, Beta y Gamma. Monografías Tercer Milenio. CONABIO, CONACYT, SEA. Vol. 4. México, D.F., México.

Chapa-Vargas, L. \& K. Monzalvo-Santos. 2012. Natural protected areas of San Luis Potosí, México: ecological representativeness, risks, and conservation implications across scales. Int. J. Geogr. Inf. Sci. Online first, DOI:10.1080/13658816.2011.643801.

CITES. 2011. Convention on International Trade in Endangered Species of Wild Fauna and Flora (CITES). Appendices I, II y III.

Colwell, R.K. 2011. EstimateS: Statistical estimation of species richness and shared species from Samples. Versión 8.2. (Consultado: 16 agosto 2011, http:// viceroy.eeb.uconn.edu/estimates).

DOF (Diario Oficial de la Federación). 2010. Norma Oficial Mexicana NOM-059-SEMARNAT-2010, Protección ambiental-Especies nativas de México de flora y fauna silvestres. Categorías de riesgo y especificaciones para su inclusión, exclusión o cambio. Lista de especies en riesgo.

Elith, J., C.H. Graham, R.P. Anderson, M. Dudi'k, S. Ferrier, A. Guisan, R.J. Hijmans, F. Huettmann, J.R. Leathwick, A. Lehmann, J. Li, L.G. Lohmann, B.A. Loiselle, G. Manion, C. Moritz, M. Nakamura, Y. Nakazawa, J. McC. Overton, A.T. Peterson, S.J. Phillips, K.S. Richardson, R. Scachetti-Pereira, R.E. Schapire, J. Soberón, S. Williams, M.S. Wisz \& N.E. Zimmermann. 2006. Novel methods improve prediction of species' distributions from occurrence data. Ecography 29: 129-151.

Escalante, P., A.G. Navarro S. \& A.T. Peterson. 1993. A geographical, ecological and historical analysis of landbird diversity in Mexico, p. 281-308. In R. Ramammorthy, R. Bye, A. Lot \& J. Fa (eds.). Biological Diversity in Mexico. Oxford University, Nueva York, Nueva York, EE.UU.

ESRI, 2008. ArcMap 9.3.License Type ArcInfo. Geographic Information System.

FAO. 2002. State of the World Forest. 2001. Food and Agriculture Organization, Rome, Italy.

Flores, O.V. \& P. Gerez. 1994. Biodiversidad y Conservación en México: vertebrados, vegetación y uso de suelo. UNAM, CONABIO, México D.F., México.

Ferrier, S. \& A. Guisan. 2006. Spatial modelling of biodiversity at the community level. J. Appl. Ecol. 43: 393-404.

García-Trejo, E.A. \& A.G. Navarro-Sigüenza. 2004. Patrones biogeográficos de la riqueza de especies y el endemismo de la avifauna en el oeste de México. Acta Zool. Mex. 20: 167-185.

González-García, F. \& H. Gómez de Silva. 2003. Especies endémicas: riqueza, patrones de distribución y retos para su conservación, p. 150-194. In H. Gómez de Silva \& A. Oliveras de Ita (eds.). Conservación de aves: experiencias en México. National Fish and Wildlife Foundation-CIPAMEX-CONABIO, México D.F., México.

Hijmans, R.J., S.E. Cameron, J.L. Parra, P.G. Jones \& A. Jarvis. 2005. Very high resolution interpolated climate surfaces for global land areas. Int. J. Climatol. 25: 1965-1978.

Howell, S.N.G. \& S. Webb. 1995. A field guide to the birds of Mexico and northern Central America. Oxford University, Nueva York, Nueva York, EE.UU.

Hutto, R.L., S.M. Pletschet \& P. Hendricks. 1986. A fixedradius point count method for nonbreeding and breeding season use. Auk 3: 593-602.

Illoldi-Rangel, P., V. Sánchez-Cordero \& A.T. Peterson. 2004. Predicting distributions of Mexican mammals using Ecological Niche Modeling. J. Mammal. 85: 658-662.

Illoldi-Rangel P., F. Trevor, M. Linaje, C. Pappas, V. Sánchez-Cordero \& S. Sarkar. 2008. Solving the maximum representation problem to prioritize areas for the conservation of terrestrial mammals at risk in Oaxaca. Divers Distrib. 14: 493-508.

INE (Instituto Nacional de Ecología). 1997. Guía de aves canoras y de ornato. INE, CONABIO, SEMARNAP, México D.F., México.

INEGI. 2003. Síntesis de Información Geográfica del Estado de San Luis Potosí. Desglose geográfico estatal. CD. México D.F., México.

Kumara, H.N., M. Irfan-Ullah \& S. Kumar, 2009. Mapping potential distribution of slender loris subspecies in peninsular India. Endang. Species Res. 7: 29-38. 
Lampila, P., M. Mönkkönen \& A. Desrochers.2005. Demographic responses by birds to forest fragmentation. Conser. Biol. 1537-1546.

Llorente-Bousquets, J. \& S. Ocegueda. 2008. Estado del conocimiento de la biota, en Capital natural de México, vol. I: Conocimiento actual de la biodiversidad. CONABIO, México D.F., México.

Luna, I., J.J. Morrone \& D. Espinosa. 2004. Biodiversidad de la Sierra Madre Oriental. Las Prensas de Ciencias. CONABIO, UNAM, México D.F., México.

Margules, C.R. \& R.L. Pressey. 2000. Systematic conservation planning. Nature 405: 242-253.

Mas, J.F., H. Puig, J.L. Palacio-Prieto \& A. Sosa. 2002. Modelado del proceso de deforestación en una región del sureste de México. CD de las memorias del II seminario Latinoamericano de Geografía Física. Maracaibo, Zulia, Venezuela.

Mittermeier, R.A. 1988. Primate diversity and the tropical forest: case studies from Brazil and Madagascar and the importance of megadiversity countries. p. 145154. In E. O. Wilson (ed.). Biodiversity.National Academy, Washington D.C., EE.UU.

National Geographic Society. 1996. Field Guide to the Birds of North America. Washington D.C., EE.UU.

Navarro, A.G., A.T. Peterson \& A. Gordillo-Martínez. 2003. Museums working together: The atlas of the birds of Mexico, p. 207-225. In N. Collar, C. Fisher \& C. Feare (eds.). Why museums matter: avian archives in an age of extinction. Bulletin British Ornithologists'Club Supplement 123A, London, England.

Navarro, S.A.G., H.A. Garza-Torres, S. López de Aquino, O.R. Rojas-Soto \& L.A. Sánchez-González. 2004. Patrones biogeográficos de la avifauna. p 439- 467. In I. Luna, J.J. Morrone \& D. Espinosa. (eds.). Biodiversidad de la Sierra Madre Oriental. Las Prensas de Ciencias. CONABIO, UNAM, México, D.F., México.

Nix, H.A. 1986. A biogeographic analysis of Australian Elapid Snakes. Atlas of Elapid Snakes of Australia, p. 4-15. In R. Longmore (ed.). Australian Flora and Fauna Series Number 7. Australian Government Publishing Service, Canberra, Sydney, Australia.

NMBCA. 2011. Acta de Conservación de Aves Migratorias Neotropicales. U.S. Fish and Wildlife Service. (Consultado: 8 agosto 2011, http:/www.fws.gov/ birdhabitat/Grants/NMBCA/BirdList.shtm).

Ortega-Huerta, M.A. \& A.T. Peterson. 2004. Modelling spatial patterns of biodiversity for conservation prioritization in North-Eastern México. Divers. Distrib. 10: $39-54$.

Ortega-Huerta, M.A. \& A.T. Peterson.2008. Modelling ecological niches and predicting geographic distributions: a test of six presence-only methods. Rev. Mex. Biodivers. 79: 205-216.

Palomera-García, C., E. Santana \& R. Amparan-Salcido. 1994. Patrones de distribución de la avifauna en tres estados del occidente de México. Anales Inst. Biol. Univ. Nac. Autón. México, Ser. Zool. 65: 137-175.

Palomera-García, C., E. Santana, S. Contreras-Martínez \& R. Amparan. 2007. Jalisco, p. 1-48. In R. OrtizPulido, A. Navarro-Sigüenza, H. Gómez de Silva, O. Rojas-Soto \& T.A. Peterson (eds.). Avifaunas estatales de México. CIPAMEX, Pachuca, Hidalgo, México.

Peterson, R.T. \& E.L. Chalif. 1989. Aves de México. Guía de Campo. Diana, México D.F., México.

Peterson, A.T. 2001. Predicting species' geographic distributions based on ecological niche modeling. Condor 103: 599-605.

Peterson, A.T., M.A. Ortega-Huerta, J. Bartley, V. SanchezCordero, J. Soberon, R.H. Buddemeier \& D.R.B. Stockwell. 2002. Future projections for Mexican faunas under global climate change scenarios. Nature 416: 626-629.

Petit, L.J. \& D.R. Petit. 2003. Evaluating the importance of human-modified lands for neotropical bird conservation. Conserv. Biol. 17: 687-694.

Phillips, R., P. Anderson \& R.E. Schapire. 2006. Maximum entropy modeling of species geographic distributions. Ecol. Model. 190: 231-259.

Ralph, C.J., G.R. Geupel, P. Pyle, T.E. Martin, D.F. De Sante \& B. Mila. 1996. Manual de métodos de campo para el monitoreo de aves terrestres. General Technical Report PSW-GTR-159. Pacific Southwest Research Station, Forest Service, U.S. Department of Agriculture, Albany, Nueva York, EE.UU.

Ramamoorthy, T.P., R. Bye, A. Lot \& J. Fa. 1993. Biological diversity of Mexico: Origins and distribution. Oxford University, Nueva York, EE.UU.

Ramírez-Albores, J.E. 2010. Diversidad de Aves de hábitats naturales y modificados en un paisaje de la Depresión Central de Chiapas, México. Rev. Biol. Trop. 58: 511-528.

Reyes, H.H., M. Aguilar, J.R. Aguirre \& J. Fortanelli.2008. Spatial Configuration of Land-use/Land-cover in the Pujal-Coy Project Area, Huasteca Potosina Region, Mexico. Ambio 5: 381-389.

Reyes-Hernandez, H., L. Olvera-Vargas, F. Sahagún-Sánchez \& J.F. Mas-Caussel. 2009. Transformation of the forest cover and future scenarios in the Sierra Madre Oriental, physiographic region, San Luis Potosí, Mexico. ISRSE 33. 33 International Symposium on Remote Sensing of Environment. Sustaining the Millennium Development Goals.

Ríos-Muñoz, C.A. \& A.G. Navarro-Sigüenza. 2009. Efectos del cambio de uso de suelo en la disponibilidad 
hipotética de hábitat para los psitácidos de México. Ornitol. Neotrop. 20: 491-509.

Ruggiero, A. \& B.A. Hawkins. 2008. Why do mountains support so many species of birds? Ecography 31 : 306-315.

Ruíz-Jiménez, C., A.O. Alcántara \& I. Luna. 2004. Límites, p. 7-24. In I. Luna, J.J. Morrone \& D. Espinosa (eds.). Biodiversidad de la Sierra Madre Oriental. Las Prensas de Ciencias. CONABIO, UNAM, México D.F., México.

Sahagún-Sánchez, F.J. 2003. Análisis de los patrones de distribución espacial de la avifauna del estado de Querétaro. Tesis de Maestría, UNAM, México D.F., México.

Sahagún-Sánchez, F.J., H. Reyes, J.L. Flores \& L. Chapa. 2011. Modelización de escenarios de cambio potencial en la vegetación y el uso de suelo en la Sierra Madre Oriental de San Luis Potosí, México. J. Lat. Am. Geogr. 10: 65-86.

Sánchez-González, L.A. \& E.A. García-Trejo. 2010. San Luis Potosí. In R. Ortiz-Pulido, A. Navarro-Sigüenza, H. Gómez de Silva, O. Rojas-Soto \& A.T. Peterson (eds.). Avifaunas estatales de México. CIPAMEX, Pachuca, Hidalgo, México.

Scachetti-Pereira, R. 2001. Desktop GARP. (Consultado: 24 mayo 2011, http://www.nhm.ku.edu/desktopgarp/ index.html).

Solano, E. \& T.P. Feria. 2007. Ecological niche modeling and geographic distribution of the genus Polianthes L. (Agavaceae) in México: using niche modeling to improve assessments of risk status. Biodivers.Conserv.16: 1885-1900.

SRTM. 2011. Shuttle Radar Topography Mission. The Mission to Map the World. (Consultado: 13 agosto 2011, http://www2.jpl.nasa.gov/srtm/index.html).

Statgraphics. 2012. Statgrraphics Centurion XVI. Software de herramientas estadísticas. Stat Point Technologies,
Inc. 1982-2011. (Consultado: 5 junio 2012, http:// www.statgraphics.net/).

Stockwell, D.R.B. \& D.P. Peters. 1999. The GARP modelling system: Problems and solutions to automated spatial prediction. Int. J. Geogr. Inform. Syst. 13: 143-158.

Stotz, D.F., J.W. Fitzpatrick, T.A. Parker III \& D.K. Moskovits. 1996. Neotropical birds. Ecology and conservation.University of Chicago, Chicago, Illinois, EE.UU.

Toledo, V.M. \&M.J. Ordóñez. 1998. El panorama de la biodiversidad de México: una revisión de los hábitats terrestres, p. 739-755. In T.P. Ramamoorthy, R. Bye, A. Lot \& J. Fa. (eds.). Diversidad biológica de México: orígenes y distribución. Instituto de Biología, UNAM. México, D.F., México.

UICN. Unión Internacional para la Conservación de la Naturaleza. 2011. Lista Roja de la Unión Internacional para la Conservación de la Naturaleza.

Van Perlo, B. 2006. Birds of Mexico and Central America. Princeton University, Princeton, Nueva Jersey, EE.UU.

Vargas-Canales, V.M. 2006. Modelaje de los patrones de riqueza y endemismo de la avifauna del estado de San Luis Potosí, México. Tesis de Licenciatura, UNAM, México D.F., México.

Villa-Bonilla, B., O. Rojas-Soto, A.G. Colodner-Chamudis \& C. Tejeda-Cruz. 2008. Inventarios municipales de avifauna y su aplicación a la conservación: El caso de Zacapoaxtla, Puebla, México. Ornitol. Neotrop. 19: 531-551.

Williams, L.G., R.H. Manson \& E. Isunza V. 2002. La fragmentación del bosque mesófilo de montaña y patrones de uso de suelo en la región oeste de Xalapa, Veracruz, México. Madera Bosques 8: 73-89.

Zar, J.H. 1999. Biostatistical Analysis. Prentice Hall, Englewood Cliffs, Nueva Jersey, EE.UU. 


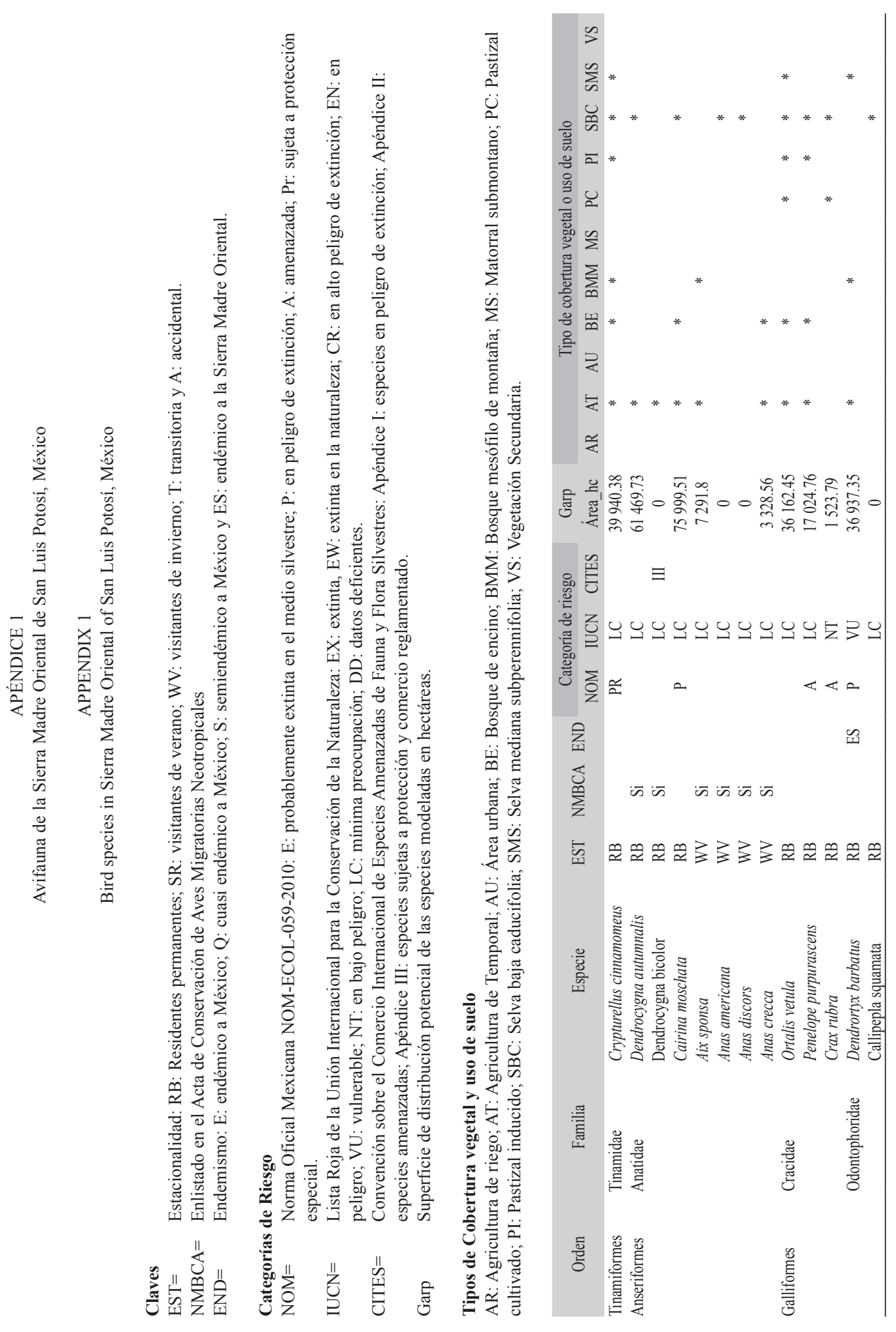




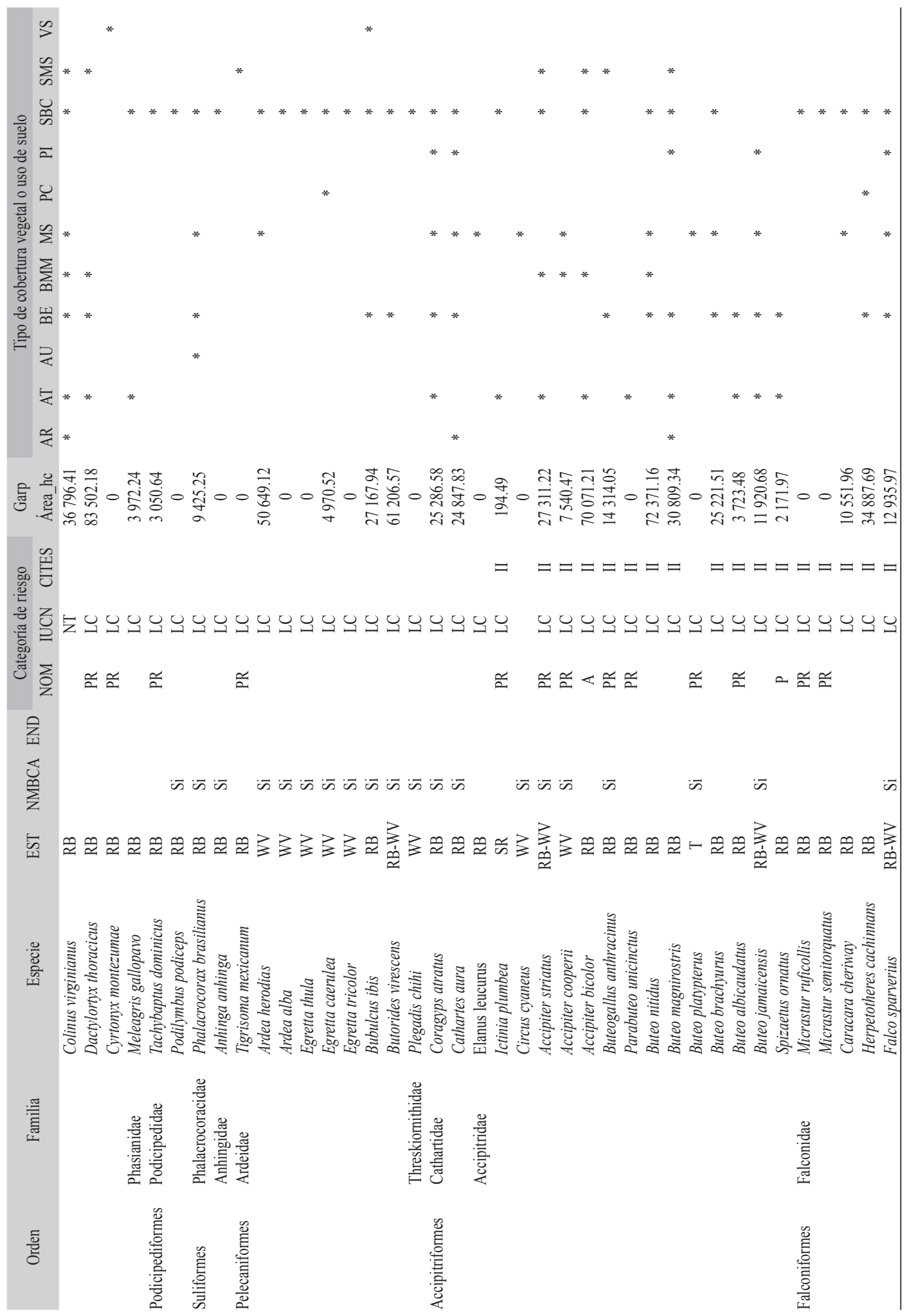




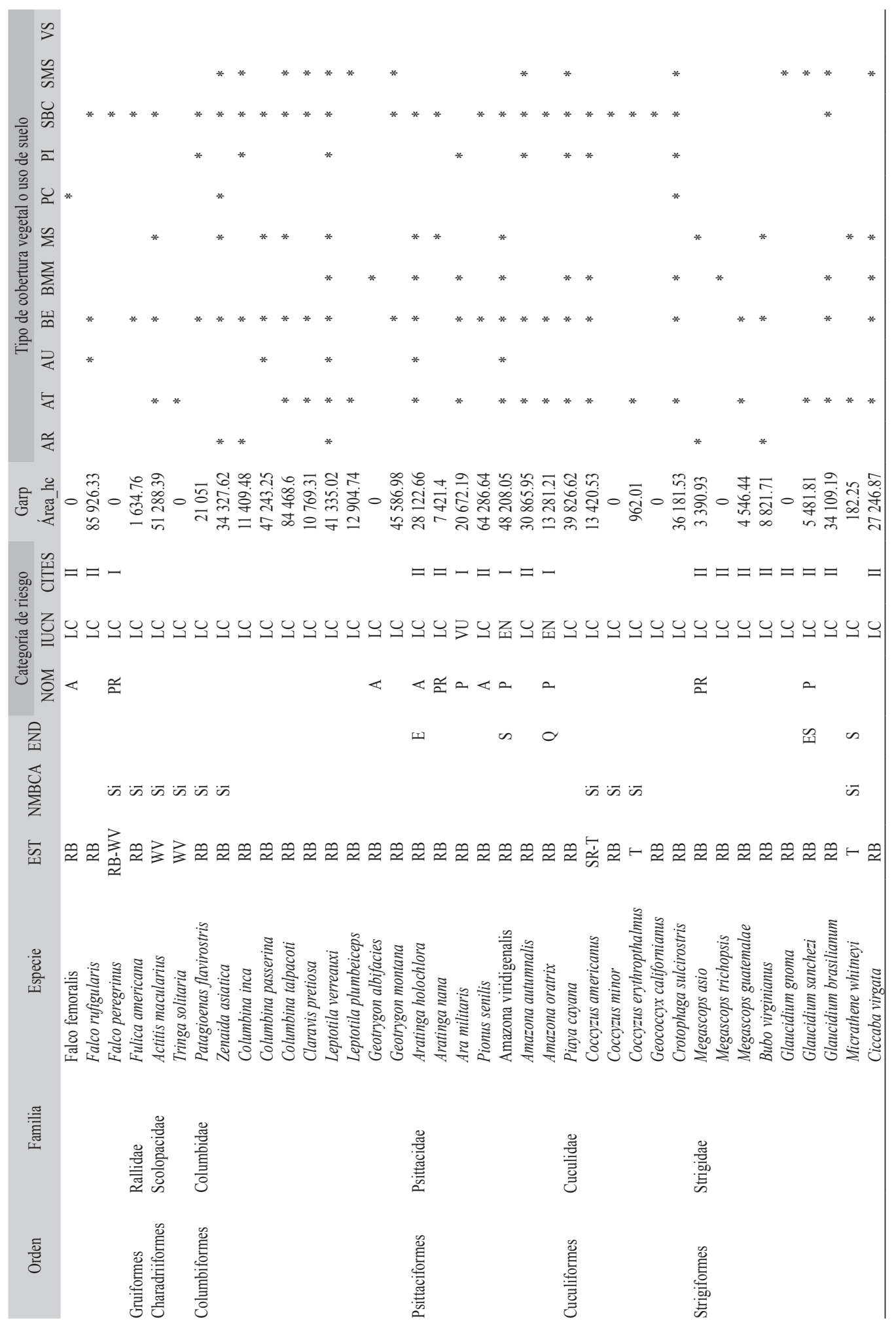




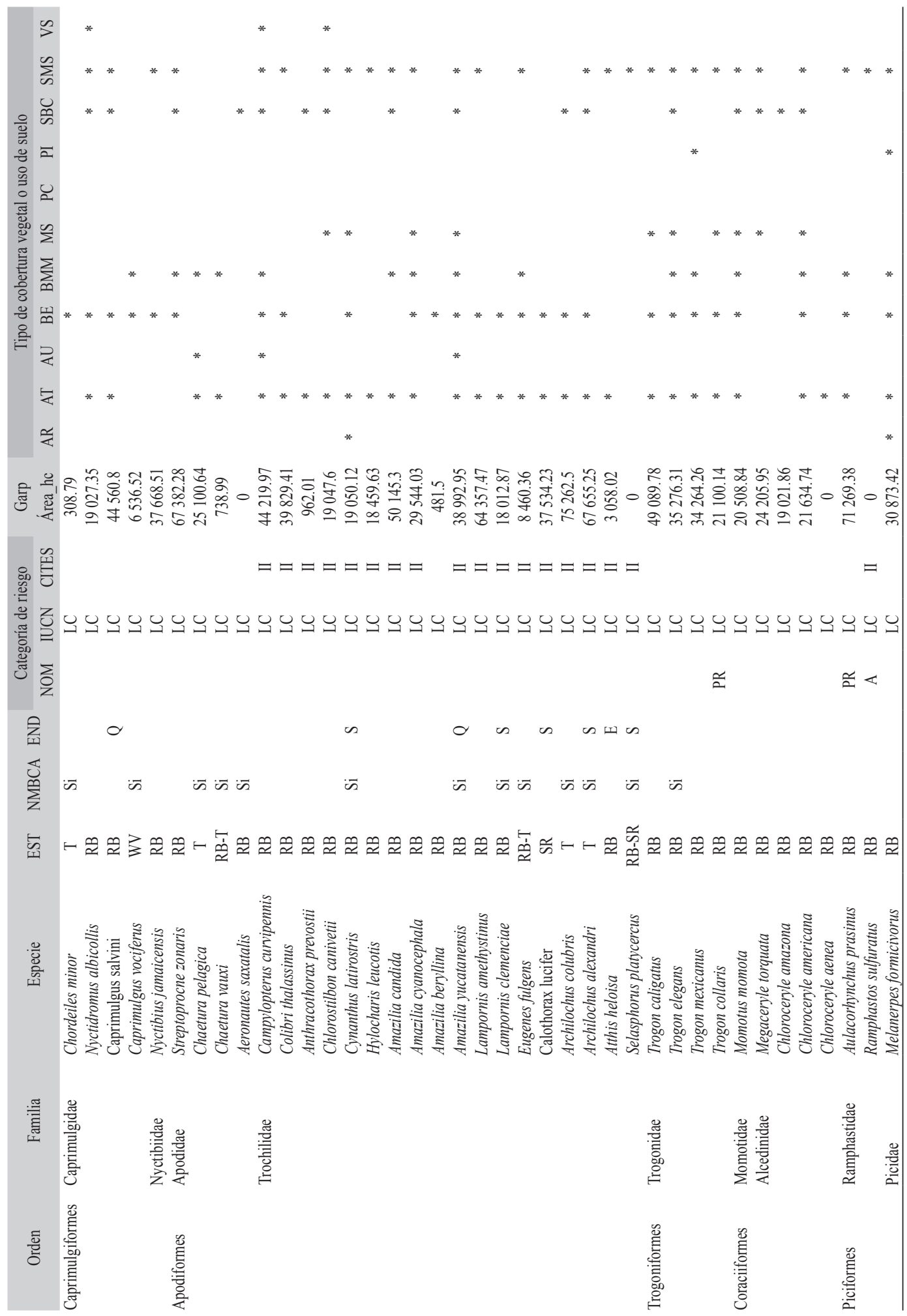




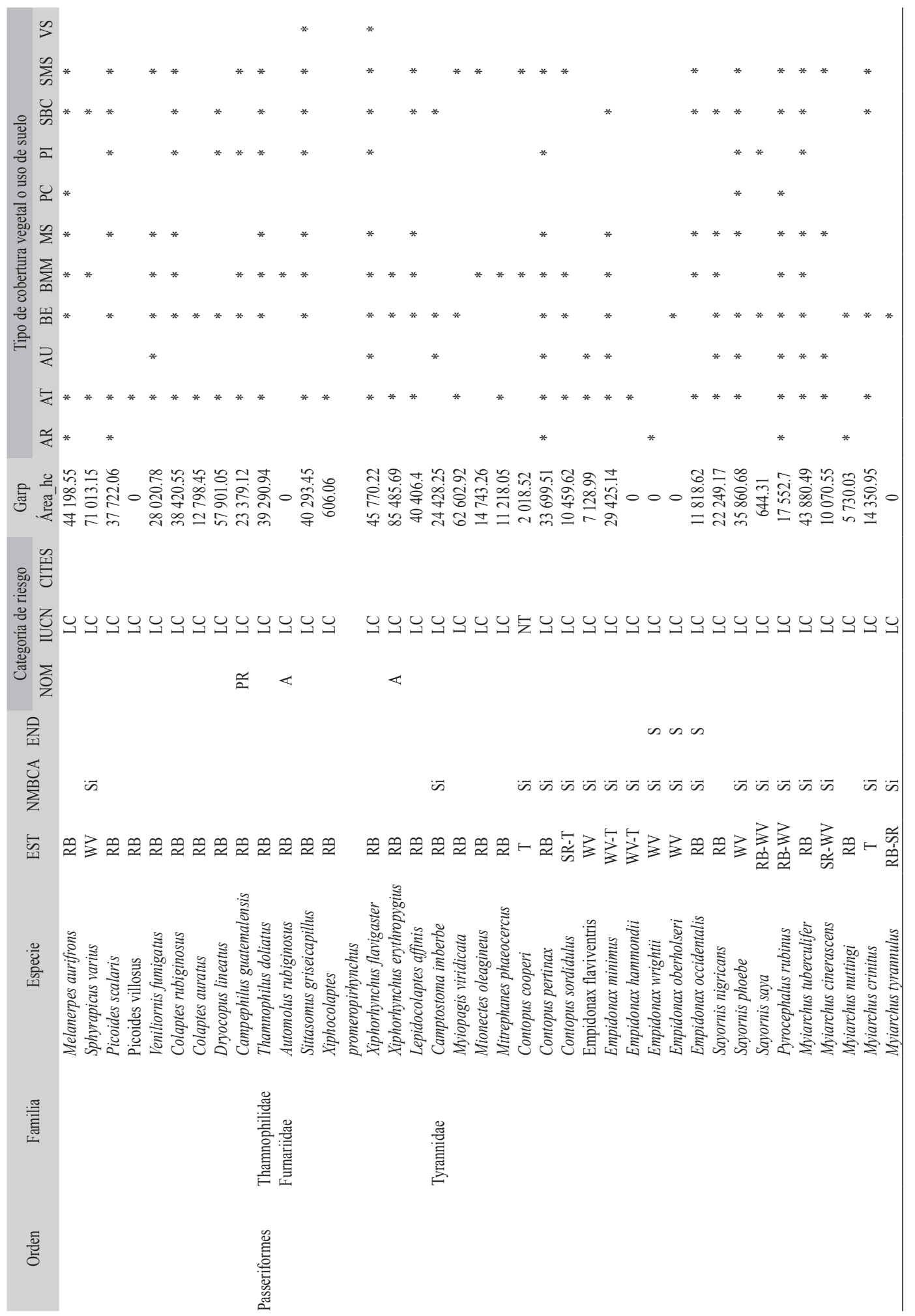




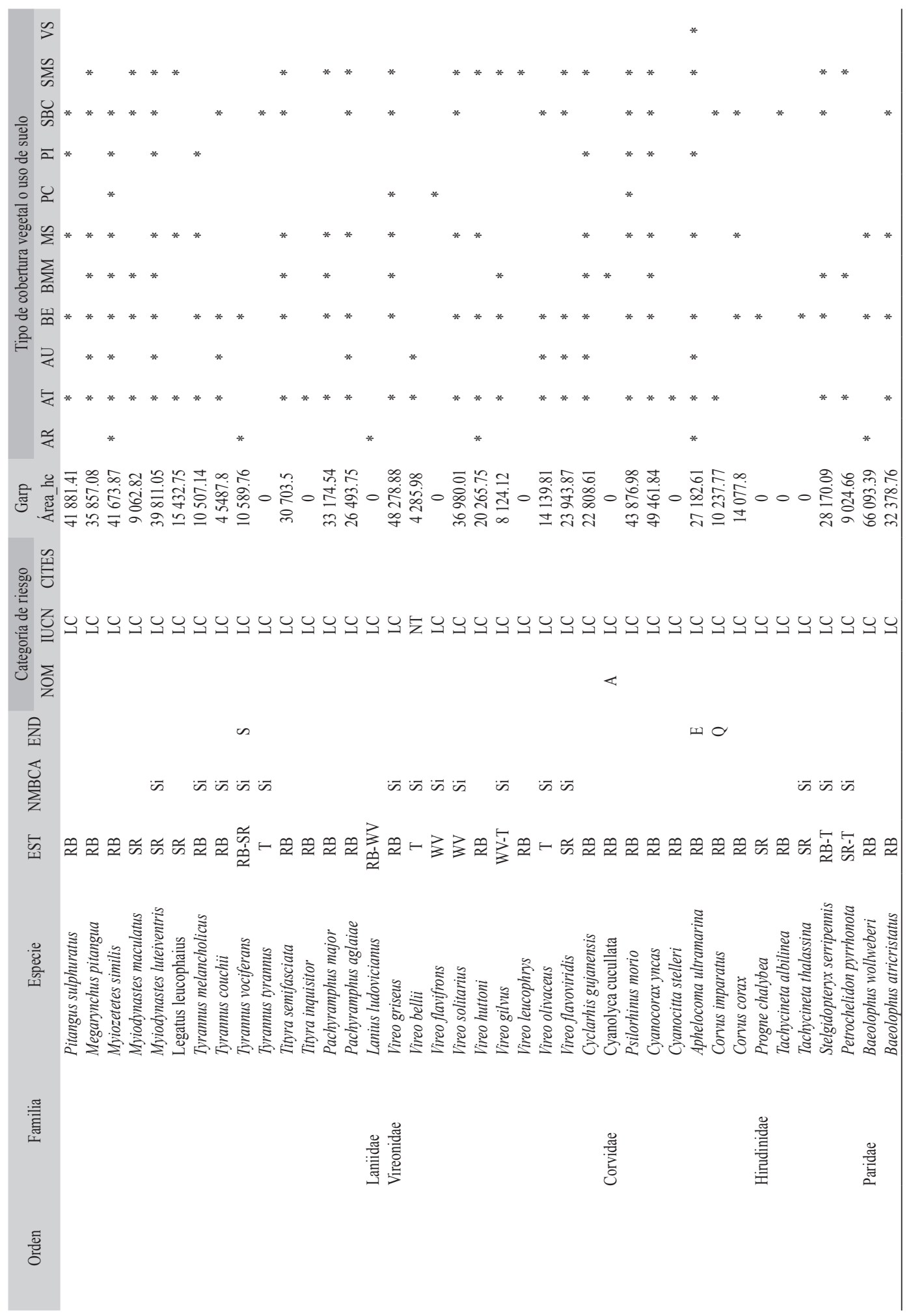




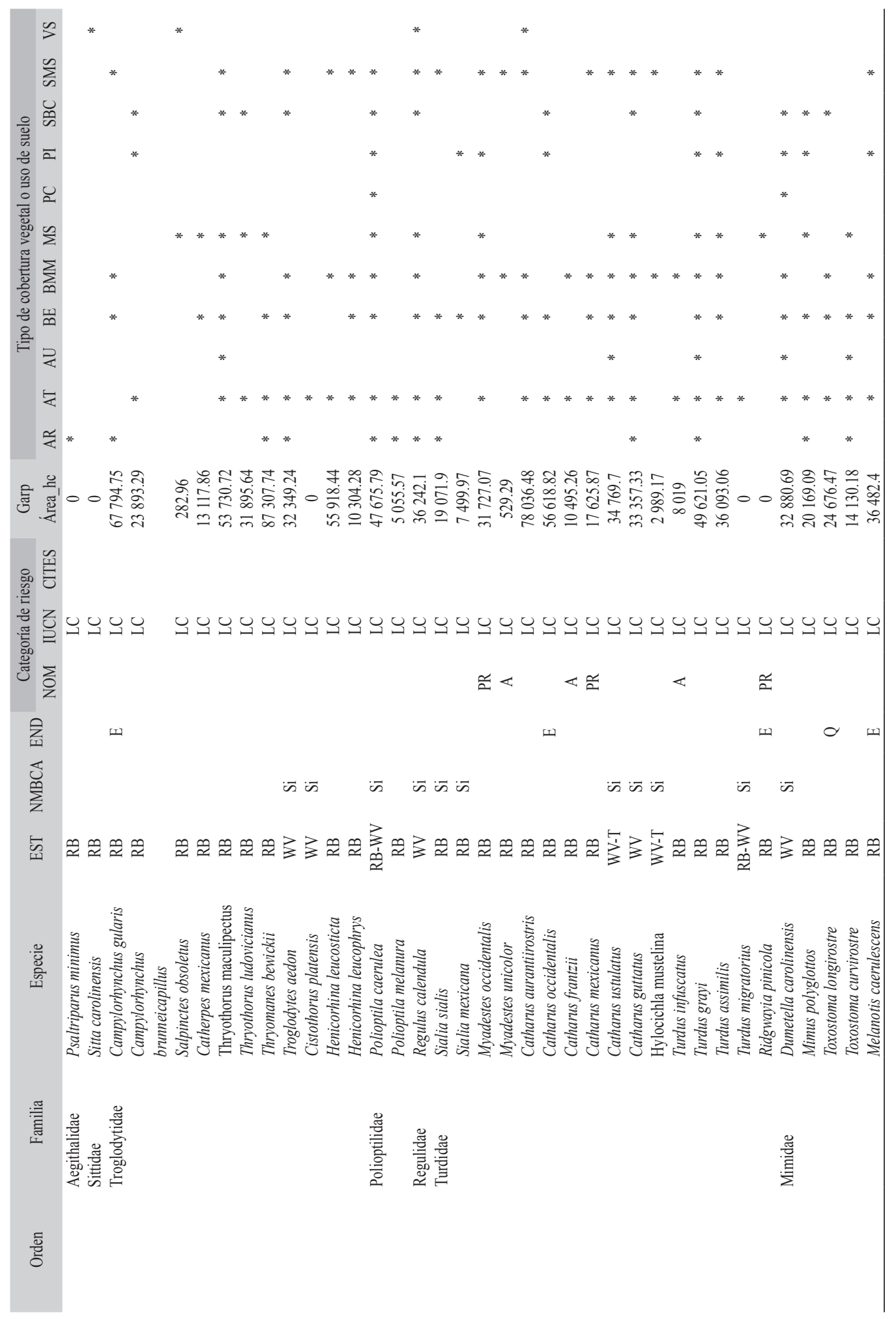




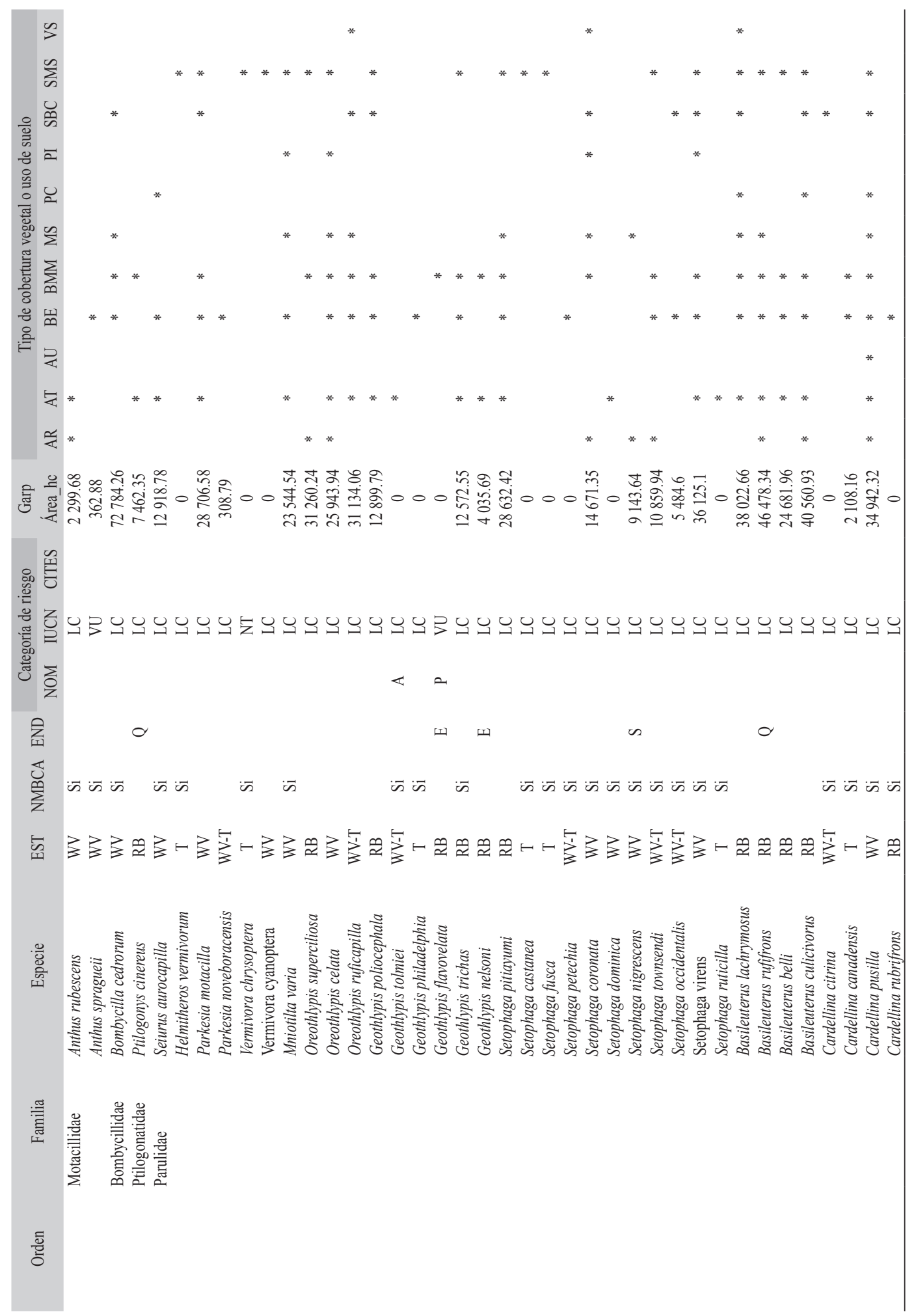




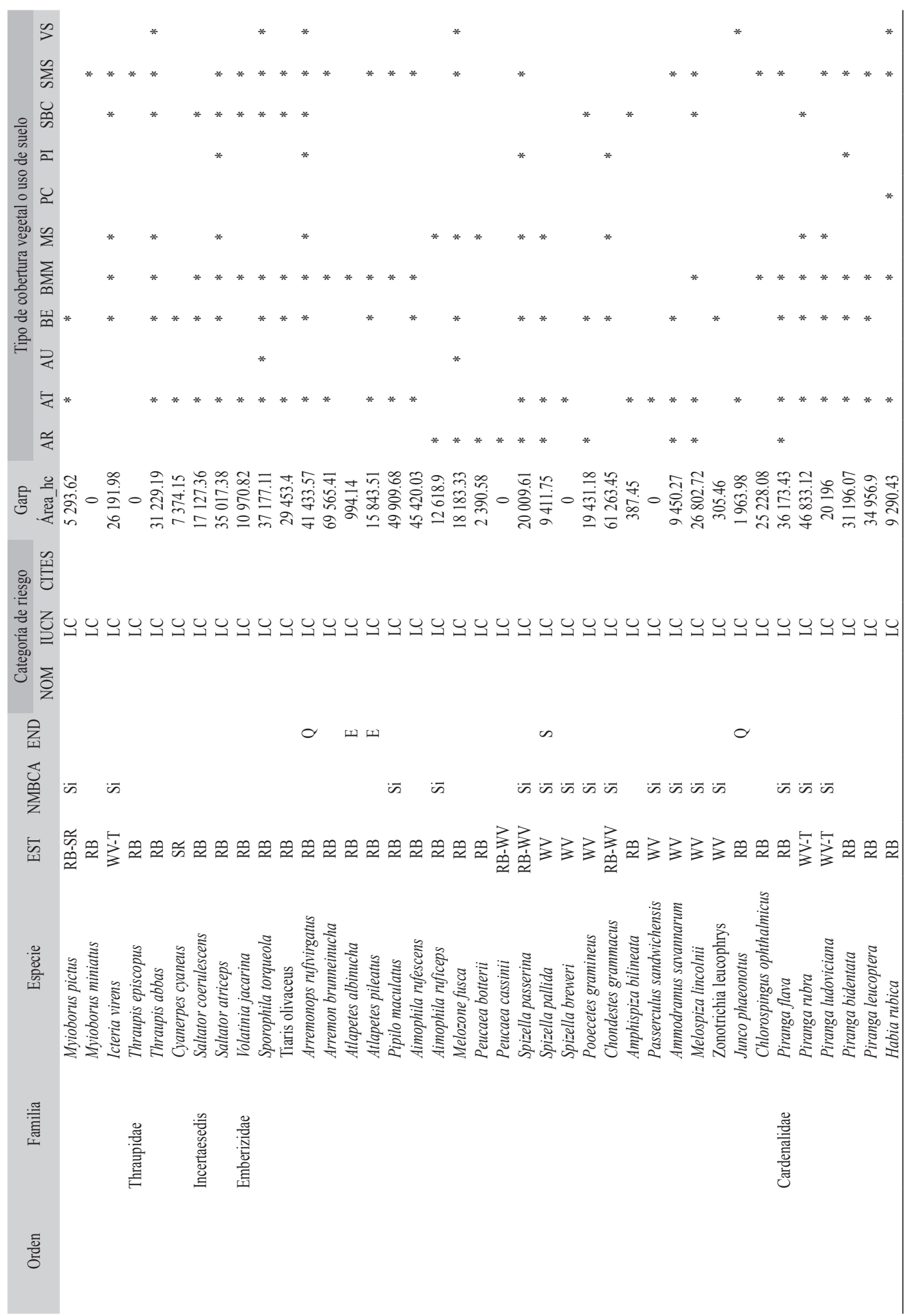




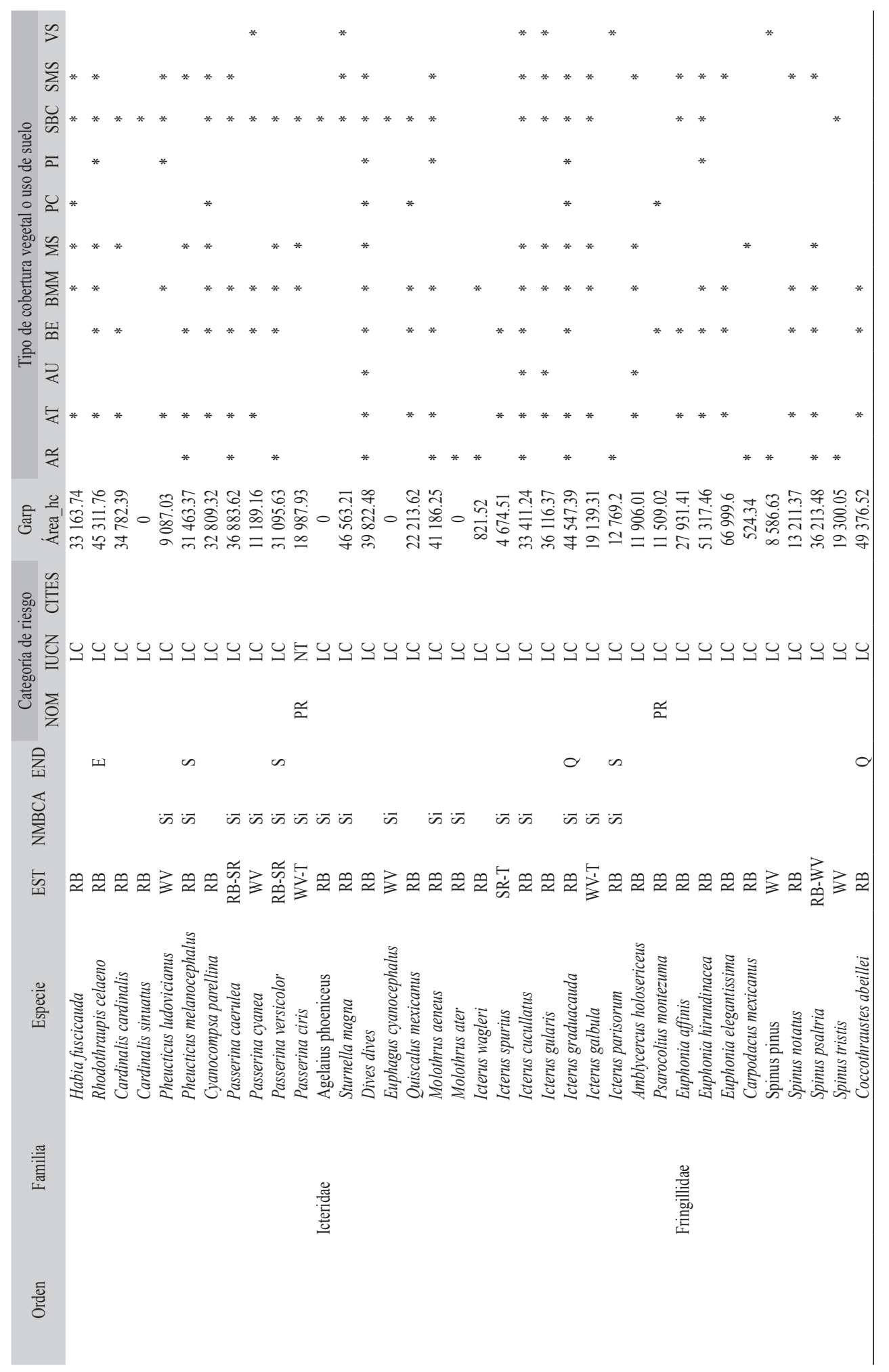


